\title{
CHARACTERIZATION OF A CONTINUOUS GEOMETRY WITHIN THE UNIT GROUP( $\left.{ }^{(}\right)$
}

\author{
BY \\ GERTRUDE EHRLICH
}

For a linear manifold whose endomorphism ring is not of characteristic 2, the projective geometry of subspaces is determined to within isomorphism or anti-isomorphism by the unit group of the endomorphism ring. The main purpose of this paper is to show that an analogous result applies in the case of von Neumann's continuous geometries and the associated continuous rings [1-7], and to give a characterization of the isomorphisms of the unit group of a continuous ring analogous to that known for the projective case.

Our treatment of the continuous case follows rather closely the treatment of the projective case given by Baer [8], but the absence of "points" (i.e., of minimal elements in the lattice of subspaces) makes it necessary in the continuous case to phrase the arguments entirely in the language of ring theory and lattice theory. The main results are contained in the Structure Theorem (Theorem 7) and the Isomorphism Theorem (Theorem 6) which we prove in Part IV. After presenting some preliminary lemmas (Part I), we discuss in Part II certain properties of two kinds of elements belonging to the unit group: involutions (elements $u$ such that $u^{2}=1$ ) and elements of class 2 (elements $t$ such that $\left.(t-1) \neq 0,(t-1)^{2}=0\right)$, and show that the elements of class 2 can be characterized within the unit group (Theorem 1). In Part III, we introduce certain sets of involutions ( $\Delta$-systems) and characterize these within the unit group (Theorem $\cdot 2$ ). We then exhibit a 1-1 correspondence between the class of all $\Delta$-systems and the class of all nonzero subspaces (Theorem 5), and show that the relation of "betweenness" in the lattice can be completely described in terms of $\Delta$-systems and certain derived systems $\Delta^{2}$ which consist of elements of class 2 (Theorem 3 ). This provides the necessary techniques for Part IV.

\section{PART I. BACKGROUND AND PRELIMINARY LEMMAS}

1. Continuous geometry; continuous ring. The subspaces of a finite dimensional projective geometry or the elements of a continuous geometry form a lattice $\mathfrak{Q}$ which is complemented, modular, complete, and topological. The

Presented to the Society, December 28, 1953 under the title Structure theorem for continuous rings; received by the editors August 23, 1954 and, in revised form, January 3, 1956.

(1) The results presented here constitute part of a doctor's dissertation written at the University of Tennessee in 1953, under the direction of Professor Wallace Givens. The new results contained in Part II were developed by Professor Givens in a seminar on continuous geometry in 1952-1953. 
range of the (normalized) dimension function $D$ defined by von Neumann on the elements $\mathfrak{a}, \mathfrak{b}, \mathfrak{c}, \cdots$ of the lattice is $\{0,1 / \nu, 2 / \nu, \cdots, 1\}$ in the case of a $(\nu-1)$-dimensional projective geometry, and $\{0 \leqq D \leqq 1\}$ in the case of a continuous geometry. For $\nu \geqq 3$, except in the case of non-Desarguesian projective planes, the lattice $\mathfrak{R}$ determines to within isomorphism a ring $\Re$ whose lattice of principal right ideals is isomorphic to $\mathbb{R}$. In the projective case, $\Re$ is isomorphic to the endomorphism ring of the linear manifold associated with the geometry. In the continuous case, $\Re$ is known as a continuous ring. In both cases, $\Re$ is an irreducible, regular ring which is complete in the topology of "rank distance," a metric related to the dimension function. "Regularity" is equivalent to the condition that every principal right ideal be generated by an idempotent. "Irreducibility" for a regular ring is equivalent to the condition that the center of the ring be a field (cf. [1-7]).

For simplicity, our results will be formulated for the continuous case only, but the arguments apply without modification to the finite-dimensional projective case.

2. Correspondence between idempotents and involutions. In relating the structure of a continuous ring $\Re$ to the structure of its unit group $B$ (the set of all nonsingular elements of $\Re$ ), a basic correspondence between the set of all idempotents $e=e^{2}$ and the set of all involutions $u=u^{-1}$ will be employed. The following lemma is readily verified.

Lemma 1. Suppose $2 \neq 0$. The mappings $u \rightarrow(1+u) / 2=e$, and $e \rightarrow 2 e-1=u$ are 1-1 inverse mappings between the set of all involutions $u$ and the set of all idempotents $e$ in $\Re$. Also, the mappings $u \rightarrow(1-u) / 2=f$ and $f \rightarrow-2 f+1=u$ are 1-1 inverse mappings.

When $2=0$, this correspondence clearly fails. The hypothesis that $\Re$ is not of characteristic 2 will therefore be maintained throughout.

3. Left-multiplications; invariant spaces. In the following, we use $(x)_{r}$ and $(x)_{l}$, respectively, to denote the principal right and left ideals generated by the ring element $x$, and refer to principal right ideals as "subspaces." The symbol $(x)_{l}^{r}$ shall denote the principal right ideal consisting of the elements $y \in \Re$ such that $x y=0$ (right annihilator of $\left.(x)_{l}\right)$; similarly, the symbol $(x)_{r}^{l}$ shall denote the principal left ideal consisting of the elements $y \in \Re$ such that $y x=0$ (left annihilator of $\left.(x)_{r}\right)$. If $t$ is any element of $\Re$, we use $\mathfrak{l}^{+}(t)$ to denote the set of all $x \in \Re$ such that $t x=x$, and $\mathfrak{l}^{-}(t)$ to denote the set of all $y \in \Re$ such that $t y=-y$. (It is obvious that $\mathfrak{I}^{+}(t)$ and $\mathfrak{I}^{-}(t)$ are the subspaces $(t-1)_{l}^{r}$ and $(t+1)_{l}^{r}$, respectively.)

Lemma 2. (a) If $u=2 e-1$ is an involution, then $\mathfrak{l}^{+}(u)=(e)_{\text {r }}$ and $\mathfrak{l}^{-}(u)$ $=(1-e)_{r}$. Also, if $v=-2 f+1$ is an involution, then $\mathfrak{l}^{+}(v)=(1-f)_{r}$ and $\mathfrak{l}^{-}(v)$ $=(f)_{r}$. (b) For any involution $u, \mathfrak{l}^{+}(u) \cup \mathfrak{l}^{-}(u)=(1)_{r}$, and $\mathfrak{l}^{+}(u) \cap \mathfrak{l}^{-}(u)=(0)_{r}$. (c) If $\mathfrak{a}$ and $\mathfrak{b}$ are two subspaces such that $\mathfrak{a} \cap \mathfrak{b}=(0)_{r}$, then there exists a unique involution $u$ such that $\mathfrak{a}=\mathfrak{l}^{+}(u)$ and $\mathfrak{b}=\mathfrak{I}^{-}(u)$. 
Proof. By [5, p. 708], (e), is the set of all $x \in \Re$ such that $e x=x$. By definition, $\mathfrak{l}^{+}(u)$ is the set of all $x \in \Re$ such that $u x=x$. If $u=2 e-1$, the conditions $e x=x$ and $u x=x$ are equivalent. Hence $\mathfrak{l}^{+}(u)=(e)_{r}$. Similarly, $\mathfrak{l}^{-}(u)=(1-e)_{r}$. Since $\mathfrak{l}^{+}(u)=\mathfrak{I}^{-}(-u)$ and $\mathfrak{l}^{-}(u)=\mathfrak{l}^{+}(-u)$, the second part of $(\mathrm{a})$ is immediate from the first part. From (a) follows (b). If $\mathfrak{a} \cup \mathfrak{b}=(1)_{r}$ and $\mathfrak{a} \cap \mathfrak{b}=(0)_{r}$, then there exists a unique idempotent $e$ such that $a=(e)_{r}$ and $\mathfrak{b}=(1-e)_{r}[5, p .708$, Lemma 3]. But then (c) is immediate by Lemma 1.

The left-multiplications in $\Re$ carry right ideals to right ideals and so induce mappings on the lattice $\mathfrak{R}$. One obtains readily:

LemMa 3. If $t$ belongs to $\Re$, the mapping $\gamma_{t}:(x)_{r} \rightarrow(t x)_{r}$ is an endomorphism of the lattice $\mathfrak{R}$ of principal right ideals of $\Re$. If $t^{-1}$ belongs to $\Re$, then $\gamma_{t}$ is an automorphism of $\mathfrak{R}$, and $\gamma_{t}(x)_{r}=\left(t x t^{-1}\right)_{r}$.

4. Center of the ring and of the unit group. It will be useful to have available certain necessary and sufficient conditions for an element of $\Re$ (or, in particular, an element of the unit group (S) to belong to the center $\mathbb{B}$ of $\Re$.

LEMMA 4. If $t$ belongs to $\mathbb{S}$, then $t$ belongs to $B$ if and only if $\gamma_{t}(x)_{r}=(x)_{r}$ for every $(x)_{r} \in \mathbb{R}$.

Proof. If $t$ belongs to $\mathbb{Z}$, then $\gamma_{t}(x)_{r}=(t x)_{r}=(x t)_{r}=(x)_{r}$ for every $(x)_{r} \in \mathbb{R}$. The converse follows in view of [2, pp. 39-44] and Lemma 3.

LEMMA 5. If $t_{1}$ and $t_{2}$ are elements of $\left(\mathbb{S}\right.$, then $\gamma_{t_{1}}=\gamma_{t_{2}}$ if and only if $t_{1}=z t_{2}$ for some $z \in \mathbb{S}$.

The proof is immediate by Lemma 4, applied to $\left(t_{2} x\right)_{r}=\left(t_{1} t_{2}^{-1} t_{2} x\right)_{r}$.

LEMMA 6. If $e$ and $f$ are idempotents and $\mathfrak{a}=(e)_{r}$, then $\mathfrak{a}=(f)_{r}$ if and only if $f=e+e y(1-e)$ for some $y \in \Re$.

This lemma is proved by von Neumann [2, p. 13, Lemma 2.7].

LEMma 7. If $t \in \Re$ commutes with every idempotent $e \in \Re$, then $t$ belongs to the center 3 of $\Re$.

Proof. Let $e=e^{2},(t)_{r}=(e)_{r}$, where $t e=e t=t$, and $e=t k$ for some $k \in \Re$. For $y \in \Re$, define idempotents $f$ and $g$ by $f=(1-e)+(1-e) y e, g=e+e k y(1-e)$. Then $(1-e) y e=f e=f t k=t f k=0 \cdot k=0$; also, by Lemma $6, t=g t$, and $0=t e k y(1-e)=e y(1-e)$. Thus $y e=e y e=e y$, and $e \in \mathbb{Z}$. Since $\Re$ is irreducible; 3 is a field, and $e=0$ or $e=1$, so that $(t)_{r}=(e)_{r}=(0)_{r}$ or $(1)_{r}$. If $(t)_{r}=(0)_{r}$, then $t=0$, and thus belongs to 3 . If $(t)_{r}=(1)_{r}$, then $t$ has an inverse in $\Re$. Since $t e=e t$ for every idempotent $e$ in $\Re, \gamma_{t}(e)_{r}=(t e)_{r}=(e t)_{r}=(e)_{r}$ for every subspace $(e)_{r}$ of $\mathfrak{R}$. But then $\gamma_{t}$ is the identity automorphism on $\mathfrak{R}$ and, by Lemma $4, t$ belongs to $\mathbb{Z}$.

By Lemma 1, it is evident that in Lemma 7, above, "idempotent" may be replaced by "involution." The next lemma then follows immediately. 
Lemma 8. The center $\overline{\mathbb{S}}$ of $B$ is the set $\overline{3}-0$ of nonzero elements in the center $\mathbb{B}$ of $\mathfrak{R}$.

5. Some further properties of idempotents. By Property 4 of the rank function [7, p. 343], any two elements $a$ and $b$ of $\Re$ which have the same rank are equivalent, i.e.: there exist $p, q \in(S)$ such that $p a q=b$. If $a=e=e^{2}$ and $b=f=f^{2}$ are two idempotents of the same rank, a stronger condition holds.

LEMMA 9. Two idempotents $e$ and $f$ of the same rank are similar, i.e., there exists an element $q \in \mathbb{B}$ such that $q e q^{-1}=f$.

Proof. Let $p$ and $q$ in $\&$ be such that $f=p e q$. Then $(f)_{r}=(p e q)_{r}=(p e)_{r}$ $=\left(p e p^{-1}\right)_{r}$. Since $p e p^{-1}$ is idempotent, Lemma 6 guarantees the existence of $x \in \Re$ such that $p e p^{-1}=f+f x(1-f)$. Let $t=1+f x(1-f)$ so that $t^{-1}=1-f x(1$ $-f)$. Then $t p e p^{-1}=(t p) e(t p)^{-1}=f$, as required.

An important class of subrings of a continuous ring $\Re$ is characterized in the following.

Lemma 10. (a) For $e \neq 0$ an idempotent belonging to $\Re$, the set $e \Re e$ of all elements exe, $x \in \Re$, is the complete, regular rank-ring corresponding to the lattice of subspaces of $(e)_{r}$.

(b) The center of eße consists of the elements in the set eße, and is isomorphic to the center $\mathbb{Z}$ of $\Re$.

Proof. (a) The regularity of $e \Re e$ is proved by von Neumann [5, p. 713]. The remaining conditions are readily deduced from von Neumann's Lemma $15.7[2$, p. 146].

(b) This was originally proved by von Neumann (unpublished).

According to von Neumann $\left[1\right.$, p. 9], a finite set of subspaces $\mathfrak{a}_{1}, \cdots, \mathfrak{a}_{k}$ is independent if and only if $\left(\mathfrak{a}_{1} \cup \ldots \cup \mathfrak{a}_{i}\right) \cap \mathfrak{a}_{i+1}=(0)_{r}, i=1, \cdots, k-1$. This condition is equivalent to $\left(\mathfrak{a}_{p_{1}} \cup \ldots \cup \mathfrak{a}_{p_{i}}\right) \cap \mathfrak{a}_{p_{i+1}}=(0)_{r}$ where $i \rightarrow p_{i}$ is any permutation of the integers $1, \cdots, k[1$, Theorem $2.2, \mathrm{p} .11]$. In this connection, we make repeated use of

LeмmA 11. If $\mathfrak{a}_{1}, \cdots, \mathfrak{a}_{k}$ are independent subspaces such that $\bigcup_{i=1}^{k} \mathfrak{a}_{i}=(1)_{r}$, then there exist idempotents $e_{1}, \cdots, e_{k}$ such that $a_{i}=\left(e_{i}\right)_{r}$ for $i=1, \cdots, k$, and $\sum_{i=1}^{k} e_{i}=1, e_{i} e_{j}=e_{j} e_{i}=0$ for $i \neq j$. (Two idempotents $e_{i}$ and $e_{j}$ with the property $e_{i} e_{j}=e_{j} e_{i}=0$ for $i \neq j$ are said to be orthogonal; $k$ mutually orthogonal $i d e m p o t e n t s$ are said to form an independent set of idempotents.)

Proof. This lemma is proved by von Neumann [2, p. 31, Lemma 3.2].

When a subspace is decomposed into $k$ independent subspaces, the following lemma is sometimes helpful:

Lemma 12. If an element $t$ of $\Re$ is expressible in the form

$$
\text { (A) }: t=\sum_{i, j=1}^{k} e_{i} x_{i j} e_{j}
$$


where $e_{i}(i=1, \cdots, k)$ form an independent set of idempotents such that $\sum_{i=1}^{k} e_{i}$ $=1$, and the $x_{i j}(i, j=1, \cdots, k)$ are elements of $\Re$, then $e_{i} x_{i j} e_{j}=e_{i} t e_{j}$ for each $i$ and $j$.

Proof. Right and left multiplication of (A) by $e_{i}$ and $e_{j}$, respectively, for each $i$ and $j$, gives the desired result.

Finally, several of our arguments will require the use of

LEMMA 13. If $g^{2}=g \neq 0$, and $k$ is an element of $\Re$ such that $g x k=0$, or $k x g=0$ for every $x \in \Re$, then $k=0$.

Proof. Suppose rank $g=\rho$, and $g x k=0$ for every $x \in \Re$. Then $x^{-1} g x k=0$ for every $x \in \mathcal{B}$, and, since $x^{-1} g x$ is an arbitrary idempotent of rank $\rho$ (Lemma 9), $\left(x^{-1} g x\right)_{l}^{r}$ is an arbitrary subspace of dimension $1-\rho$. Hence $(k)_{r}$ is contained in every subspace of dimension $1-\rho$.

If $k \neq 0,(k)_{r}$ contains a subspace $\mathfrak{a} \neq(0)$, whose dimension is less than or equal to $\rho \neq 0$. If $\mathfrak{b}$ is any complement of $\mathfrak{a}$, then $\mathfrak{b}$ has dimension greater than or equal to $1-\rho$. Hence $\mathfrak{b}$ contains a subspace of dimension $1-\rho$, and we have $\mathfrak{b} \supseteq \mathfrak{c} \supseteq(k)_{r} \supseteq \mathfrak{a}$. But $\mathfrak{b} \supseteq \mathfrak{a}$ is clearly impossible for $\mathfrak{a} \neq(0)_{r}$. Hence $k=0$. An analogous argument applied to the lattice of left ideals again gives the conclusion $k=0$ when $k x g=0$ for all $x$.

\section{PART II. InVOLUTIONS AND ELEMENTS OF CLASS 2}

\section{First and second centralizer of an involution.}

Definition 1 . If $\mathfrak{S}$ is a set of elements of $\mathbb{S}$, then the set $\mathfrak{S}(\mathfrak{S})$ of all elements of $\$ S$ which commute with every element of $\mathfrak{S}$ is said to be the (first) centralizer of $\mathfrak{S}$. (In particular, if $\mathfrak{S}$ consists of a single element $t$, the set $\mathfrak{S}(t)$ of all elements of $\$(S)$ which commute with $t$ is said to be the (first) centralizer of $t$.)

The centralizer of the centralizer $\mathbb{S}(\mathfrak{S})$ of a set $\mathbb{S}$ of elements of $\mathbb{S}$ is referred to as the second centralizer $\mathfrak{S}^{2}(\mathfrak{S})$ of the set $\mathfrak{S}$. It is evident that the centralizer of any subset of $B$ is a subgroup of $\mathbb{B}$. Also, the following relations are easily seen to hold: 1 . For $\mathfrak{S}_{1} \subseteq \Im_{2}, \mathfrak{S}\left(\Im_{2}\right) \subseteq \mathbb{S}\left(\Im_{1}\right)$. 2. For any element $s \in \mathbb{G}, \mathbb{S}^{2}(s) \subseteq \mathbb{S}(s)$.

Proposition 1. If $t$ is an element of $\mathfrak{(}(u)$, and $u$ is an involution, the following conditions are equivalent:

(a) $t$ belongs to the centralizer of $u$,

(b) $e t(1-e)=(1-e)$ te for $e=(1+u) / 2$,

(c) $\gamma_{\mathfrak{t}} \mathfrak{l}^{+}(u)=\mathfrak{l}^{+}(u)$ and $\gamma_{\mathfrak{t}} \mathfrak{l}^{-}(u)=\mathfrak{l}^{-}(u)$,

(d) $t=e x e+(1-e) y(1-e)$ for some $x, y \in \Re$.

Proof. The equivalence of (a) and (b) is readily verified. Lemma 2 gives the equivalence of (a) and (c). From $t=e t e+e t(1-e)+(1-e) t e+(1-e) t(1-e)$ it follows that (b) implies (d), while (b) follows from (d) on left and right multiplication by $e$. 
Proposition 2. An element $s$ of \& belongs to the second centralizer $\mathbb{S}^{2}(u)$ of an involution $u$ if and only if $s=z_{1}+z_{2}(1-e)$ for $e=(1+u) / 2, z_{1}, z_{2} \in \mathfrak{Z}$.

Proof. If $s$ belongs to $\mathfrak{f}^{2}(u)$, then by Proposition $1 \mathrm{~d}$ and Lemma 12 , $s=e s e+(1-e) s(1-e)$. From this, we have $(e s e)(e x e)=(e x e)(e s e)$ for arbitrary $x \in \Re$, so that ese belongs to the center of $e \Re e$. By Lemma 10, ese $=z_{1} e$ for some $z_{1} \in Z$. Similarly, $(1-e) s(1-e)=z_{2}(1-e)$ for some $z_{2} \in Z$, and $s=z_{1} e+z_{2}(1-e)$, as required. The converse follows directly.

Lemma 14. (a) A subspace $\mathfrak{a}$ is invariant under $\gamma_{u}$ for $u$ an involution if and only if $\mathfrak{a}=\left[\mathfrak{a} \cap \mathfrak{l}^{+}(u)\right] \cup\left[\mathfrak{a} \cap \mathfrak{l}^{-}(u)\right]$.

(b) If a subspace $a$ is invariant under $\gamma_{u}$ for every involution $u \in \Re$, then $\mathfrak{a}=(0)_{\text {, }}$ or $\mathfrak{a}=(1)_{\text {r. }}$.

Proof. (a) Let $u=2 e-1$, and let $\mathfrak{a}=(a)_{r}$ for some $a \in \Re$. Suppose $\gamma_{\boldsymbol{u}} \mathfrak{a}$ $=((2 e-1) a)_{r}=(a)_{r}=a$. Then $(2 e-1) a=a x$ for some $x \in \Re$, so that $e a$ $=1 / 2[a(1+x)]=a y$ for some $y \in \Re$. Also, $(1-e) a=a-e a=a-a y=a(1-y)$. Now, $(a)_{r}=([e+(1-e)] a)_{r}=(e a+(1-e) a)_{r} \subseteq(e a)_{r} \cup((1-e) a)_{r}$. But $(e a)_{r}$ $=(a y)_{r} \subseteq(e)_{r} \cap(a)_{r}=\mathfrak{a} \cap(e)_{r}$. Similarly, $((1-e) a)_{r} \subseteq \mathfrak{a} \cap(1-e)_{r}$. Hence $\mathfrak{a}$ $=(a)_{r} \subseteq\left[\mathfrak{a} \cap(e)_{r}\right] \cup\left[\mathfrak{a} \cap(1-e)_{r}\right]=\left[\mathfrak{a} \cap \mathfrak{l}^{+}(u)\right] \cup\left[\mathfrak{a} \cap \mathfrak{l}^{-}(u)\right] . \quad$ Trivially, $\left[\mathfrak{a} \cap \mathfrak{l}^{+}(u)\right] \cup\left[\mathfrak{a} \cap \mathfrak{l}^{-}(u)\right] \subseteq \mathfrak{a}$, and we have the equality.

(b) Suppose $\mathfrak{a} \neq(0)_{r}, \neq(1)_{r}$, and let $\mathfrak{b}$ be a complement of $\mathfrak{a}$. Then $\mathfrak{b} \neq(1)_{r}$, $\neq(0)_{r}$, and $\mathfrak{a}$ is a complement of $\mathfrak{b}$. By the irreducibility of $\mathfrak{R}$, there exists another complement $\mathfrak{a}^{\prime}$ of $\mathfrak{b}$ such that $\mathfrak{a}^{\prime} \neq \mathfrak{a}$. Since $\operatorname{dim} \mathfrak{a}=\operatorname{dim} \mathfrak{a}^{\prime}, \mathfrak{a}^{\prime} \neq \mathfrak{a}$ implies $\mathfrak{a}^{\prime} \nsupseteq \mathfrak{a}$. But then $(\mathfrak{a} \cap \mathfrak{b}) \cup\left(\mathfrak{a} \cap \mathfrak{a}^{\prime}\right)=(0)_{r} \cup\left(\mathfrak{a} \cap \mathfrak{a}^{\prime}\right) \subseteq \mathfrak{a}$.

Let $u$ be the involution such that $\mathfrak{l}^{+}(u)=\mathfrak{b}, \mathfrak{l}-(u)=\mathfrak{a}^{\prime}$ (Lemma 2). Then, by (a), a cannot be invariant under $\gamma_{u}$, contrary to hypothesis. It follows that $\mathfrak{a}=(0)_{r}$ or $\mathfrak{a}=(1)_{r}$.

Proposition 3. If $\mathfrak{a}$ is a subspace such that $\gamma_{\mathfrak{v}} \mathfrak{a}=\mathfrak{a}$ for every involution $v$ in the centralizer $\mathbb{S}(u)$ of some involution $u$, then either $\mathfrak{a}=(0)_{r}$, or $\mathfrak{a}=\mathfrak{l}^{+}(u)$, or $\mathfrak{a}=\mathfrak{I}-(u)$, or $\mathfrak{a}=(1)$.

Proof. Since $u$ belongs to $\mathfrak{(}(u)$, we have, in particular, $\gamma_{u} \mathfrak{a}=\mathfrak{a}$. Hence, by Lemma $14 \mathrm{a}, \mathfrak{a}=\left[\mathfrak{a} \cap(e)_{r}\right] \cup\left[\mathfrak{a} \cap(1-e)_{r}\right]$, where $e=(1+u) / 2$. Since the mapping $(x)_{r} \rightarrow \gamma_{v}(x)_{r}\left((x)_{r}\right.$ arbitrary in $\left.\mathfrak{R}\right)$ is a lattice automorphism, $\gamma_{\mathfrak{v}} \mathfrak{a}$ $=\gamma_{v}\left[\mathfrak{a} \cap(e)_{r}\right] \cup_{\gamma_{v}}\left[\mathfrak{a} \cap(1-e)_{r}\right]$. By Lemma $1, v \in \mathbb{S}(u)$ implies $v=e v e+(1$ $-e) v(1-e)$. Hence $\gamma_{v}(e)_{r}=(v e)_{r}=(e v e)_{r} \subseteq(e)_{r}, \quad$ and $\gamma_{v}(1-e)_{r}=(v(1-e))_{r}$ $=((1-e) v(1-e))_{r} \subseteq(1-e)_{r}$. The existence of $v^{-1}$ implies equality in each case. Hence

$$
\gamma_{v}\left[\mathfrak{a} \cap(e)_{r}\right]=\gamma_{v} \mathfrak{a} \cap \gamma_{v}(e)_{r}=\mathfrak{a} \cap(e)_{r}
$$

and

$$
\gamma_{v}\left[\mathfrak{a} \cap\left(1-e_{r}\right)\right]=\gamma_{v} \mathfrak{a} \cap \gamma_{v}(1-e)_{r}=\mathfrak{a} \cap(1-e)_{r} .
$$

From $v^{2}=1$ and $e v=v e$, we have $(e v e)^{2}=e,[(1-e) v(1-e)]^{2}=1-e$. Hence 
eve and $(1-e) v(1-e)$ are involutions relative to the subrings $e \Re e$ and $(1-e) \Re(1-e)$, respectively. By Lemma 10 , these are the regular rings corresponding, respectively, to the lattice of subspaces of $(e)_{r}$ and the lattice of subspaces of $(1-e)_{r}$. If we let $\mathfrak{a} \cap(e)_{r}=(e k)_{r}$, and $\mathfrak{a} \cap(1-e)_{r}=((1-e) h)_{r}$ for some $h, k \in \Re$, then $\gamma_{v}\left[\mathfrak{a} \cap(e)_{r}\right]=\gamma_{v}(e k)_{r}=(e k)_{r}=(v e k)_{r}=(e v e e k)_{r}=\gamma_{\text {eve }}(e k)$ $=\mathfrak{a} \cap(e)_{r}$. Similarly, $\gamma_{(1-e) v(1-e)}(1-e)_{r}=\mathfrak{a} \cap(1-e)_{r}$. Since $v$ is an arbitrary involution in $\Re$, eve and $(1-e) v(1-e)$ are arbitrary involutions in $e \Re e$ and $(1-e) \Re(1-e)$, respectively. But then $\mathfrak{a} \cap(e)_{r} \subseteq(e)_{r}$ is invariant under every


Similarly, $\mathfrak{a} \cap(1-e)_{r}=(1-e)_{r}$ or $(0)_{r}$. Since $\mathfrak{a}=\left[\mathfrak{a} \cap(e)_{r}\right] \cup\left[\mathfrak{a} \cap(1-e)_{r}\right]$, we have either $\mathfrak{a}=(0)_{r}$, or $\mathfrak{a}=(e)_{r}=\mathfrak{l}^{+}(u)$, or $\mathfrak{a}=(1-e)_{r}=\mathfrak{l}-(u)$, or $\mathfrak{a}=(1)_{r}$ as required.

\section{Elements of class 2.}

Definition 2. An element $s$ of $\Re$ is said to be of class $i$ if $i$ is the smallest integer for which $(s-1)^{i}=0$ (cf. [8, p. 207]).

We consider here the elements $s$ for which $i=2$, and prove first a number of lemmas.

LEMMA 15. (a) Every element of class 2 belongs to (\$), and can be represented in the form $1+e x(1-e)$ for some $x \in \Re, e=e^{2} \in \Re$. (b) If $s$ belongs to $\Re$ and $s=1+e x(1-e)$ for $x \in \Re$ and $e$ an idempotent such that $e x(1-e) \neq 0$, then $s$ is of class 2. (c) The set of elements of class 2 in $\Re$ is nonempty.

Proof. A straightforward argument gives (a) and (b). By Lemma 13, if $e x(1-e)=0$ for every $x \in \Re$, every $e=e^{2} \in \Re$, then $e=0$ or $e=1$ for every idempotent $e \in \Re$. But then $\Re$ is a division ring, contrary to the hypothesis that $\Re$ is a continuous ring. This establishes (c).

LEMMA 16. If $s=1+n$ is of class 2 , the following conditions hold: (a) $\mathfrak{l}+(s)$ $=(n)_{l}^{r}$. (b) If a is a subspace comparable with $(n)_{r}$, then $a$ is invariant under $\gamma_{s .}$.

Proof. From the definition of $\mathfrak{l}^{+}(s)$, (a) follows immediately. Suppose $\mathfrak{a} \subseteq(n)_{r}$. Since $n^{2}=0,(n)_{r} \subseteq(n)_{l}^{r}$, and (a) implies that the subspace $\mathfrak{a}$ is elementwise invariant under $\gamma_{s}$. If $\mathfrak{a} \supseteq(n)_{r}$ and $e$ is an idempotent such that $(n)_{r}=(e)_{r}$, then, by the modular law, $\left[(e)_{r} \cap(1-e)_{r}\right] \cup \mathfrak{a}=(e)_{r} \cup\left[\mathfrak{a} \cap(1-e)_{r}\right]$. If $\mathfrak{c}=\mathfrak{a} \cap(1-e)_{r}$ and $\mathfrak{b}$ is a relative complement of $\mathfrak{c}$ in $(1-e)_{r}$, then $\mathfrak{c}, \mathfrak{b}$ and $(e)_{r}$ are independent subspaces, and an idempotent $g$ may be chosen so that $\mathfrak{c}=(g)_{r}$ and $(e)_{r} \cup \mathfrak{b}=(1-g)_{r}$. From $(g)_{r} \subseteq(1-e)_{r}$ and $(e)_{r} \subseteq(1-g)_{r}$, it follows that $e g=g e=0$. But then $\mathfrak{a}=(e)_{r} \cup(g)_{r}=(e+g)_{r}$, and $\gamma_{\mathfrak{a}} \mathfrak{a} \subseteq(e)_{r} \cup(g)_{r}=\mathfrak{a}$.

LEMMA 17. If $s=1+n$ is an element of class 2 , and $\mathfrak{b}$ is a complement of $(n)_{l}^{r}$, then (a) $\gamma_{1+n} \mathfrak{b} \cup \mathfrak{b}=(n)_{r} \cup \mathfrak{b}$, and (b) $\gamma_{1+n} \mathfrak{b} \cap \mathfrak{b}=(0)_{r}$.

Proof. The proof of (a) is straightforward. To obtain (b), we observe that the dimension of $\left[\gamma_{1+n} \mathfrak{b} \cup \mathfrak{b}\right]$ is twice the dimension of $\mathfrak{b}$. From this it follows that $\gamma_{1+n} \mathfrak{b} \cap \mathfrak{b}$ has dimension zero and is therefore equal to $(0)_{r}$. 


\section{First and second centralizer of a class 2 element.}

Proposition 4. Given $s=1+n, n \neq 0, n^{2}=0$, and $e, f$ and $g$ three mutually orthogonal idempotents such that $(e)_{r}=(n)_{r},(f)_{r}$ is a relative complement of $(e)_{r}$ in $(n)_{l}^{r}$, and $(g)_{r}$ is a complement of $(n)_{l}^{r}$. Then an element $t$ of $B$ is contained in the centralizer $\mathbb{E}(s)$ of $s$ if and only if $t=e x_{1} e+e x_{2} f+e x_{3} g+f x_{4} f+f x_{5} g+r^{-1}\left(e x_{1} e\right) r$ where $x_{1}, \cdots, x_{5}$ are elements of $\Re$, and $r$ is an element of $\&$ satisfying the conditions (a) $n r^{-1}=e$, (b) $e r=n$, and (c) $r g=g$.

Proof. Suppose $t$ belongs to $\mathfrak{S}(s)$. From $(n)_{r}=(e)_{r} \subseteq(n)_{l}^{r}$, we have $n=n(e+f+g)=n g$. By Lemma $8 \mathrm{~b}, \gamma_{1+n}(g)_{r} \cap(g)_{r}=(g+n g)_{r} \cap(g)_{r}=(0)_{r}$. If $x$ and $y$ are such that $(g+n g) x=g y$, then $g y=(1+n) g x=g x=0$. Hence $n x$ $=g(y-x)$ for some $x$ and $y$ implies $g x=0$. In particular, for $x=y$, we obtain (A): If $n x=0$ for some $x$, then $g x=0$. Also, the following statements are easily derived. (B): If $x n=0$ for some $x$, then $x e=0$. (C):fte $=0$. (D): gte $=0$. (E): $g t f=0$.

Since $e$ and $g$ are of equal rank, Lemma 9 requires the existence of $p \in(B)$ such that $e p^{-1}=p^{-1} g$. If $r=g p e+f+n$, then $r^{-1}=e p^{-1}+f+g k e$, where $k$ is such that $e=n k$. Hence $r$ belongs to (B). One readily verifies that $r$ satisfies conditions (a), (b) and (c) of the theorem, and that $g t g=r^{-1}(e t e) r$. From $t=(e+f+g) t(e+f+g)$, employing Lemma 12 and relations (C), (D) and (E) above, one obtains the desired representation of $t$.

The proof of the converse is trivial.

Proposition 5. If $s=1+n$ is of class 2 , then an element $d$ of $(5)$ belongs to the second centralizer $\mathcal{S}^{2}(s)$ if and only if $d=z_{1}+z_{2} n$ for some $z_{1}, z_{2} \in 3$.

Proof. Suppose $d$ belongs to $\mathfrak{S}^{2}(s)$. Then $d$ belongs to $\mathfrak{S}(s)$, and $d=e d e$ $+e d f+e d g+f d f+f d g+r^{-1}(e d e) r$, where $e, f$ and $g$ are as defined in Proposition 4 , and $r$ may be taken to be $g p e+f+n$ as in the proof of Proposition 4. If $t_{1}=e x_{1} e+f+r^{-1}\left(e x_{1} e\right) r$ for $e x_{1} e$ an arbitrary element with inverse $e y_{1} e$ relative to $e$ in $e \Re e$ (cf. Lemma 10), then $r^{-1}\left(e x_{1} e\right) r$ belongs to $g \Re g$, and has the inverse $r\left(e y_{1} e\right) r_{-1}$ one relative to $g$. Hence $t_{1}$ has the inverse $e y_{1} e+f+r\left(e y_{1} e\right) r^{-1}$ and is an element of $B$.

By Proposition $4, t_{1}$ belongs to $\mathfrak{C}(s)$, and $d$ commutes with $t_{1}$. It follows that $e d e$ commutes with $e x_{1} e$ for $e x_{1} e$ an arbitrary element with inverse relative to $e$ in $e \Re e$. By Lemma 8, applied to the ring $e \Re e$ (cf. Lemma 10), ede belongs to the center of $e \Re e$, and hence, by Lemma 10, $e d e=e z_{1} e=z_{1} e$ for some $z_{1} \in \mathcal{Z}$.

From $1=r^{-1} r=r^{-1} g p+r^{-1} f+r^{-1} n$ and $r^{-1} n=1-r^{-1} g p-r^{-1} f=1-e-f=g$, it follows that $r^{-1}(e d e) r=z_{1} g$. Hence $d=z_{1}+d_{1}$ where $d_{1}=e d f+e d g+f\left(d-z_{1}\right) f$ $+f d g$ or, by Lemma $12, d_{1}=e d_{1} f+e d_{1} g+f\left(d_{1}-z_{1}\right) f+f d_{1} g=(e+f) d_{1}(f+g)$. Imposing consecutively the conditions that $d_{1}$ commutes with $t_{2}=1+f x g$ and $t_{3}=1+\operatorname{exf}$ (both of which belong to $\mathbb{S}(s)$ ), and again with $t_{1}$, we obtain that $e x_{1} e$ commutes with $e d_{1} g r^{-1}$. Since $g r^{-1}+g k e$ (with $k$ as defined in the proof of Proposition 4), $e d_{1} g r^{-1}$ is an element of $e \Re e$. Hence, since $e x_{1} e$ is an arbitrary 
invertible element of $e \Re e$, it follows by Lemmas 8 and 10 that $e d g r^{-1}=z_{2} e$ for some $z_{2} \in Z$. But then $e d_{1} g=z_{2} e r=z_{2} n$, and $d=z_{1}+z_{2} n$, as required.

The proof of the converse is trivial.

9. Multiplicative characterization of class 2 elements. The elements of class 2 can now be characterized entirely within the unit group (5).

TheOREM 1 (Grvens). An element $s$ of $B$ is of class 2 if and only if it has the following properties:

(1) If $t$ is an element of $\mathbb{G}$, then $\mathbb{S}(t)$ properly contains $\mathfrak{S}(s)$ if and only if $t$ is in the center $\overline{\mathbb{3}}$ of $\mathbb{B}$ (i.e., if and only if $\mathbb{S}(t)=(\mathfrak{S})$.

(2) There exists an involution u such that usu $=s^{-1}$.

(3) There exists an element $r$ belonging to $\mathfrak{S}^{2}(u)$ such that $r s r^{-1}=s^{2}$.

(4) $s^{3} \neq 1$ except in the case when all elements $x$ of \&s satisfying (1), (2) and (3) have $x^{3}=1$.

Proof. To prove the necessity of each of the four conditions, set $s=1+n$ for $n \neq 0, n^{2}=0$.

(1) If $t$ belongs to $\overline{\mathbb{Z}}$, then $\mathfrak{S}(t)=\mathfrak{S} \supseteq \mathfrak{S}(s)$. But $\mathfrak{S}(t)=\mathfrak{S}(s)=\mathbb{S}$ is impossible since $s \in \mathbb{Z}$ implies that the nilpotent element $n$ belongs to the field $\mathbb{Z}$ and is therefore equal to zero, contrary to hypothesis. Hence $\mathbb{}(t) \supset \mathbb{}(s)$. Conversely, if $\mathbb{S}(t) \supset \mathbb{S}(s)$, then $t \in \mathbb{S}^{2}(s)$ and, by Proposition $5, t=z_{1}+z_{2} n$. If $z_{2} \neq 0$, then $\mathfrak{S}(t)=\mathbb{S}(s)$, contrary to hypothesis. Hence $z_{2}=0$, and $t=z_{1} \in \mathfrak{Z}$.

(2) Take $u=2 e-1$ for $e=e^{2}$ such that $n=e x(1-e)$. Then $u$ has the required property: $u s u=(2 e-1)[1+e x(1-e)](2 e-1)=1-e x(1-e)=s^{-1}$.

(3) Take $r=1+e$ for $e=(1+u) / 2$, $e$ such that $n=e x(1-e)$. By Proposition $2, r$ belongs to $\mathbb{S}^{2}(u)$. Since $r^{-1}=1-e / 2, r s r^{-1}=1+2 e x(1-e)=s^{2}$.

(4) Clearly, $s^{3}=(n+1)^{8}=1$ if and only if $3 n=0$. From $n \neq 0$ follows $3=0$ and hence $s^{3}=1$ for all elements of class 2 .

This concludes the proof of the necessity of (1)-(4). To prove the sufficiency, assume that an element $s$ of $B$ satisfies conditions (1)-(4). Let $t=s+s^{-1}$. Then (a) $t \in(B)$, (b) $t \in \bar{B}$, (c) $t^{2}-t-2=0$. For, by (2), there exists an involution $u$ such that $u s^{-1}=s u$, and $u \in \mathfrak{E}(t)$. By (3), there exists $r$ in $\mathbb{E}^{2}(u)$ such that $r s r^{-1}=s^{2}$. Since $r \in \mathbb{S}^{2}(u)$, let $r=z_{1}+z_{2}(1-e)$ for $e=(1+u) / 2$, $z_{1}, z_{2} \in 3$. From $u t=t u$ follows $r t=t r$; also, $r u=u r$. Hence $t=r t r^{-1}=r\left(s+s^{-1}\right) r^{-1}$ $=t^{2}-2$, and $t^{2}-t-2=0$. But then $t$ has the inverse $(t-1) / 2$ and belongs to 5 .

Clearly, $\mathfrak{S}(t) \supseteq \mathfrak{E}(s)$. By (2), there exists an involution $u$ such that $u s u$ $=s^{-1}$. Then $u\left(s+s^{-1}\right) u=s^{-1}+s$, or $u t=t u$, and $u \in \mathbb{C}(t)$. But $u$ does not belong to $\mathfrak{C}(s)$; otherwise, $u s u=s^{-1}=s$ would imply $s=1$, so that $\mathfrak{E}(s)=\mathbb{B}$. Thus $\mathfrak{C}(x)$ cannot properly contain $\mathfrak{S}(s)$ for any $x$, in contradiction to (1). Hence $\mathfrak{S}(t) \supset \mathfrak{S}(s)$. By (1), it follows that $t$ belongs to $\overline{\mathfrak{z}}$. But then $(t-2)(t+1)$ $=0$ implies $t=2$ or $t=-1$. If $s+s^{-1}=t=2$, then $(s-1)^{2}=0$ and, in view of (1), $s$ is of class 2 . If $t=-1$, then $s^{8}=1$. This contradicts (4) unless every element of $(5)$ which satisfies (1), (2), and (3) has $s^{8}=1$. Since all the elements of class 2 necessarily satisfy (1), (2) and (3), this is the case in which $1+3 n=1$ for every 
$n \neq 0$ such that $n^{2}=0$, or $3 n=0$ for every $n$ with this property. (By Lemma 15 c, elements $n \neq 0$ such that $n^{2}=0$ actually do exist in $\Re$.) But $3 n=0, n \neq 0$ is precisely equivalent to $3=0$, or: $2=-1$. Hence $s+s^{-1}=2$ implies, as before, that $(s-1)^{2}=0$ and, since $s \neq 1, s$ is of class 2 . This completes the proof.

10. Connection between involutions and class 2 elements.

Proposition 6. An element $t$ of (s) is of class 2 if and only if it is the product of two distinct involutions $u$ and $v$ such that $\mathfrak{l}^{+}(u)=\mathfrak{l}^{+}(v)\left(\right.$ or $\left.\mathfrak{l}^{-}(u)=\mathfrak{l}^{-}(v)\right)$.

Proof. Let $u$ and $v$ be two distinct involutions such that $\mathfrak{l}^{+}(u)=\mathfrak{l}^{+}(v)$ and let $u=2 e-1, v=2 f-1$. By Lemma 2 and Lemma $6,(e)_{r}=(f)_{r}$ and $f$ $=e+e x(1-e)$ for some $x \in \Re$. Hence $u v=1-e \cdot 2 x(1-e)$. From $u \neq v, e \neq f$, it follows that $e x(1-e)$ is different from zero, so that $u v$ is of class 2 . Conversely, suppose $s$ is of class 2 . Let $s=1+n$, with $n \neq 0, n^{2}=0$. By Lemma $15, n$ $=e y(1-e)$ for some $e=e^{2}$, some $y \in \Re$. Let $y=2 x$, and let $f=e+e x(1-e)$. Then the involutions $u=2 e-1$ and $v=2 f-1$ have the required properties. (The alternative assertion of the proposition is obtained from $(-u)(-v)$ $=u v$, and $\left.\mathfrak{l}^{+}(-u)=\mathfrak{I}^{-}(u), \mathfrak{l}^{+}(-v)=\mathfrak{l}^{-}(v)\right)$.

\section{Part III. Cosets of Involutions}

Definition 3. For $\mathfrak{a}$ a subspace belonging to $\mathfrak{l}$, let $\Delta(\mathfrak{a})^{+}$denote the set of all involutions $u$ for which $\mathfrak{a}=\mathfrak{l}^{+}(u)$ and let $\Delta(\mathfrak{a})$ - denote the set of all involutions $v$ for which $\mathfrak{a}=\mathfrak{l}^{-}(v)$. Then $\Delta(\mathfrak{a})^{+}$and $\Delta(\mathfrak{a})^{-}$will be referred to as $\Delta$-sets, and the pair $\left[\Delta(\mathfrak{a})^{+}, \Delta(\mathfrak{a})^{-}\right]$will be referred to as a $\Delta$-system (cf. $[8$, p. 219]).

Definition 4. As usual, the normalizer of a set $\phi$ of elements of $\$$ will be the set $\psi$ of elements of $\mathbb{B}$ such that for every $u \in \phi, x \in \psi$, there exists $v \in \phi$ for which $u x=x v$. The set of involutions in the normalizer of $\phi$ is denoted by $N \phi$.

11. Properties of $\Delta$-sets.

Proposition 7. If $\phi$ is a $\Delta$-set, then $\phi$ has the following properties:

(a) If $u, v, w$ are elements of $\phi$, then $u v w=w v u$ is an element of $\phi$.

(b) For every $u, v$ in $\phi$, there exists a unique $w$ in $\phi$ such that wow $=u$.

(c) An involution $u$ belongs to $N \phi$ if and only if there exists an element $w$ in $\phi$ such that $u w=w u$.

(d) If $t$ is an element of $\phi^{2}$, then $t$ is of class 1 or of class 2 .

Proof. Suppose $\phi=\Delta(\mathfrak{a})^{+}$for some subspace $\mathfrak{a}$. (If $\phi=\Delta(\mathfrak{a})$ - for some subspace $a$, then $-\phi=\Delta(\mathfrak{a})^{+}$. Since the four conditions (a)-(d) still apply if the sign of every involution in $\phi$ is changed, no separate proof will be needed for this case.) (a) If $u, v, w$ belong to the $\Delta$-set $\phi$, then $\mathfrak{I}^{+}(u)=\mathfrak{l}^{+}(v)=\mathfrak{l}^{+}(w)$. If $e, f$ and $g$ are idempotents such that $u=2 e-1, v=2 f-1$ and $w=2 g-1$, then, by Lemma $2,(e)_{r}=(f)_{r}=(g)_{r}$. Using the relations $e f=f, f g=g$ and $e g=g$, we obtain $u v w=u-v+w$. (A). Similarly, $w v u=w-v+u=u-v+w$, so that 
$u v w=w v u=(u v w)^{-1}$ is an involution. If $x$ belongs to $\mathfrak{l}^{+}(u)=\mathfrak{l}^{+}(v)=\mathfrak{l}^{+}(w)$, then $x$ belongs to $\mathfrak{l}^{+}(u v w)$. Conversely, if $x$ belongs to $\mathfrak{l}^{+}(u v w)=(e g-e f+e)_{r}$, then $x$ belongs to $(e)_{r}=\mathfrak{l}^{+}(u)=\mathfrak{l}^{+}(v)=\mathfrak{l}^{+}(w)$. Hence $\mathfrak{l}^{+}(u v w)=\mathfrak{l}^{+}(u)=\mathfrak{l}^{+}(v)=\mathfrak{l}^{+}(w)$ and $u v w$ belongs to the $\Delta$-set $\phi$.

(b) Let $w=(u+v) / 2$. Then $w$ has the required properties. (By Proposition $6, w^{2}=1$, and it is easily verified that $w$ belongs to $\phi$ and $w v w=u$.) Formula (A) gives the uniqueness.

(c) For $n \in N \phi, w \in \phi$, there exists $w^{*}$ in $\phi$ such that $w^{*}=u w u$. By (b), there exists a unique $v \in \phi$ such that $u w u=v w v$. By formula (A), $v$ commutes with $u$ as required. Conversely, suppose $u v=v u$ for some involution $u$ and some $v$ in $\phi$. Let $v=2 f-1$. If $w$ belongs to $\phi$, then, by Lemma $6, w=2 f+2 f x(1$ $-f)-1$ for some $x$ in $\Re$. Let $w^{*}=2 f+2 f u x u(1-f)-1$; then $w^{*} u=u w$, so that $u$ belongs to $N \phi$.

(d) If $t$ is an element of $\phi^{2}$, then $t=u v$ for $u, v \in \phi$, and $\mathfrak{l}^{+}(u)=\mathfrak{l}^{+}(v)$. If $u \neq v$, then $t$ is of class 2, by Proposition 6. If $u=v, t$ is obviously of class 1 .

Lemma 18. If $w$ is an involution, and $a, b$ are arbitrary in $\Re$, then $w a=b w$ implies (a) $\gamma_{w} \mathfrak{l}^{+}(a)=\mathfrak{l}^{+}(b)$ and (b) $\gamma_{w} \mathfrak{l}^{-}(a)=\mathfrak{l}^{-}(b)$. The proof is straightforward.

Propositron 8. If a set $\phi$ of involutions has properties (a) to (d) of Proposition 7, then (1) $\phi^{2}$ is an abelian subgroup of (S); (2) aua $=a^{-1}$ for every $u \in \phi$, $a \in \phi^{2}$; (3) If $\phi$ contains at least two involutions, then $\phi \subseteq \Delta\left[\mathfrak{l}^{+}\left(\phi^{2}\right)\right]+$ or $\phi$ $\subseteq\left[\mathfrak{l}^{+}\left(\phi^{2}\right)\right]^{-}$, where $\mathfrak{l}^{+}\left(\phi^{2}\right)$ denotes the intersection of all $\mathfrak{l}^{+}(t)$ for $t \in \phi^{2}$.

Proof. With the aid of Proposition 7a, (1) and (2) are easily established. (3) If $\phi$ contains at least two involutions, then $\mathfrak{l}^{+}\left(\phi^{2}\right) \neq(0)_{r}, \neq(1)_{r}$. For, let $y$ belong to $\phi^{2}, y=1+n$ for some $n$ such that $n^{2}=0$. Then $\mathfrak{I}^{+}(y)=(n)_{l}^{r}$ and $\mathfrak{l}^{+}\left(\phi^{2}\right)=\bigcap_{1+n \in \phi^{2}}(n)_{l}^{r}$. Since $\phi^{2}$ is a group, $\left(1+n_{1}\right)\left(1+n_{2}\right)$ belongs to $\phi^{2}$ for any $1+n_{1}, 1+n_{2}$ in $\phi^{2}$. By Proposition $7(\mathrm{~d}),\left[\left(1+n_{1}\right)\left(1+n_{2}\right)-1\right]^{2}=0$. Since $\phi^{2}$ is abelian, $n_{1} n_{2}=n_{2} n_{1}$, and this reduces to $n_{1} n_{2}=0$. Hence $U_{1+n \in \phi^{2}}(n)_{r}$ $\subseteq \bigcap_{1+n \in \phi^{2}}(n)_{2}^{r}$. But $\phi$ contains at least two involutions. Hence some $n$ is not zero. (The pairs $1,-1 ; 1, u ;-1, u$ cannot constitute $\phi$, by Proposition 7(d); and, for $u \neq 1, v \neq 1, u v=1$ would imply $u=v$.) It follows that $\bigcap_{1+n \in \phi^{2}}(n)_{l}^{r}$ $=\mathfrak{l}^{+}\left(\phi^{2}\right) \neq(0)_{r}$.

Also, $\mathfrak{l}^{+}\left(\phi^{2}\right)$ is not equal to (1) ${ }_{r}$. Otherwise, Proposition $7(\mathrm{~d})$ would require 1 to be the only element in $\phi^{2}$, contrary to hypothesis. Now, $\mathfrak{l}^{+}\left(\phi^{2}\right)$ is invariant under $\gamma_{t}$ for every involution $t$ in $N(\phi)$. For, clearly, $N \phi \subseteq N \phi^{2}$, and, corresponding to any $p \in \phi^{2}$ there exists $q=q(p) \in \phi^{2}$ such that $t p=q t$. Hence, by Lemma $18, \gamma_{\mathfrak{l}} \mathfrak{l}^{+}(p)=\mathfrak{l}^{+}(q)$ for all $p \in \phi^{2}$, all $q=q(p) \in \phi^{2}$, and we have

$$
\bigcap_{p \in \phi^{2}} \gamma_{\mathfrak{l}} \mathfrak{l}^{+}(p)=\gamma_{\mathfrak{t}} \bigcap_{p \in \phi^{2}} \mathfrak{l}^{+}(p)=\bigcap_{q=q(p) \in \phi^{2}} \mathfrak{l}^{+}(q) \subseteq \mathfrak{l}^{+}\left(\phi^{2}\right) .
$$

Since $\operatorname{dim} \gamma_{\mathfrak{l}} \mathfrak{l}^{+}\left(\phi^{2}\right)=\operatorname{dim} \mathfrak{l}^{+}\left(\phi^{2}\right)$, it follows that $\operatorname{dim}$

$$
\bigcap_{q=q(p) \in \phi^{2}} \mathfrak{l}^{+}(q)=\operatorname{dim} \mathfrak{l}^{+}\left(\phi^{2}\right) \text {, }
$$


and $\cap \mathfrak{l}^{+}(q)=\mathfrak{l}^{+}\left(\phi^{2}\right)$. Thus $\gamma_{t} \mathfrak{l}^{+}\left(\phi^{2}\right)=\mathfrak{l}^{+}\left(\phi^{2}\right)$ for every $t$ belonging to $N \phi$. If $u$ belongs to $\phi$, and $s$ is an arbitrary involution in the centralizer $\mathbb{S}(u)$ of $u$, then Proposition 7 (c) requires that $s$ belong to $N \phi$. Hence $\mathfrak{I}^{+}\left(\phi^{2}\right)$ is invariant under $\gamma_{s}$ for every involution in the centralizer of $u$. By Proposition 3, $\mathfrak{l}^{+}\left(\phi^{2}\right)$ is either $(0)_{r}, \mathfrak{l}^{+}(u), \mathfrak{l}^{-}(u)$, or $(1)_{r}$. But we have shown that $\mathfrak{l}^{+}\left(\phi^{2}\right)$ is different from $(0)_{r},(1)_{r}$. Hence either $\mathfrak{l}^{+}\left(\phi^{2}\right)=\mathfrak{l}^{+}(u)$ or $\mathfrak{l}^{+}\left(\phi^{2}\right)=\mathfrak{l}^{-}(u)$.

Suppose $\mathfrak{I}^{+}\left(\phi^{2}\right)=\mathfrak{l}^{+}(u)$ for some $u \in \phi$. Then $\mathfrak{l}^{+}\left(\phi^{2}\right)=\mathfrak{l}^{+}(v)$ for every $v \in \phi$. For, given $v$ an arbitrary element of $\phi$, there exists $w$ in $\phi$ such that $v=w u w$, or $v w=w u$ (Proposition 7b). By Lemma 18, $\mathfrak{l}^{+}(v)=\gamma_{w} \mathfrak{l}^{+}(u)=\gamma_{w} \mathfrak{l}^{+}\left(\phi^{2}\right)$. But $w \in \phi$ implies $w \in N \phi$. Hence $\gamma_{w} \mathfrak{l}^{+}\left(\phi^{2}\right)=\mathfrak{l}^{+}\left(\phi^{2}\right)$, and $\phi \subseteq \Delta\left[\mathfrak{l}^{+}\left(\phi^{2}\right)\right]^{+}$. Similarly, if $\mathfrak{I}^{-}(u)=\mathfrak{l}^{+}\left(\phi^{2}\right)$ for some $u \in \phi$, then $\mathfrak{I}^{-}(v)=\mathfrak{l}^{+}\left(\phi^{2}\right)$ for every $v \in \phi$, and $\phi \subseteq \Delta\left[\mathfrak{l}^{+}\left(\phi^{2}\right)\right]^{-}$.

Corollary 1. (a) If $(0)_{r} \subset \mathfrak{a} \subseteq(1)_{r}$, then $\mathfrak{a}=\mathfrak{l}^{+}[\Delta(\mathfrak{a})]^{2}$ (where $\mathfrak{l}^{+}[\Delta(\mathfrak{a})]^{2}$ $\left.=\mathfrak{I}^{+}\left[\Delta(\mathfrak{a})^{+}\right]^{2}=\mathfrak{l}^{+}\left[\Delta(\mathfrak{a})^{-}\right]^{2}\right)$.

(b) If $(0)_{r} \subset \mathfrak{a}=(e)_{r}$, then $\mathfrak{a}=\mathfrak{l}^{+}[\Delta(\mathfrak{a})]^{2}=\bigcap_{t \in \Delta(a) 2} \mathfrak{l}^{+}(t)=\bigcap_{(o)_{r}} \subseteq \mathfrak{a}[\operatorname{ex}(1-e)]_{\mathfrak{l}}^{r}$.

Proof. (a) If $\phi=\Delta(\mathfrak{a})^{+}$, and $\phi$ contains at least two involutions, then Propositions 7 and 8 require that $\phi \subseteq \Delta\left[\mathfrak{l}^{+}\left(\phi^{2}\right)\right]+$ or $\phi \subseteq \Delta\left[\mathfrak{l}^{+}\left(\phi^{2}\right)\right]^{-}$. If $\phi \subseteq \Delta\left[\mathfrak{l}^{+}\left(\phi^{2}\right)\right]^{-}$and $u$ and $v$ are any two distinct involutions in $\phi$ then $\mathfrak{l}^{+}(u)$ $=\mathfrak{l}^{+}(v)=\mathfrak{a}$, and $\mathfrak{l}^{-}(u)=\mathfrak{l}^{-}(v)=\mathfrak{l}^{+}\left(\phi^{2}\right)$. But then, by Lemma $2, u=v$, contrary to hypothesis. Hence $\phi \subseteq \Delta\left[\mathfrak{l}^{+}\left(\phi^{2}\right)\right]^{+}$and, if $u$ is any involution in $\phi=\Delta(\mathfrak{a})^{+}$, then $\mathfrak{a}=\mathfrak{l}^{+}(u)=\mathfrak{l}^{+}\left(\phi^{2}\right)$. Similarly, if $\phi=\Delta(\mathfrak{a})^{-}$, and $\phi$ contains at least two involutions, then $\phi \subseteq \Delta\left[\mathfrak{l}^{+}\left(\phi^{2}\right)\right]^{-}$, and again $\mathfrak{a}=\mathfrak{l}^{+}\left(\phi^{2}\right)$. If $\phi$ contains only one involution $u$, and $\phi=\Delta(e)_{r}^{+}$for the idempotent $e=(1+u) / 2$, then for $e \neq 0$, $e \neq 1$, Lemma 13 requires that there exist $x \in \Re$ such that $f=e+e x(1-e)$. But then, by Lemma $6,(e)_{r}=(f)_{r}$, and therefore $\mathfrak{l}^{+}(u)=\mathfrak{l}^{+}(v)$ for $v=2 f-1, v \neq u$, so that $v$ belongs to $\Delta(e)_{r}^{+}$, contrary to hypothesis. It follows that $e=0$ or $e=1$, and $u=-1$ or $u=1$. But if $\phi=1$, then $\phi=\Delta(1)_{r}^{+}, \mathfrak{a}=(1)_{r}=\mathfrak{l}^{+}(1)^{2}=\mathfrak{I}^{+}(1)$; and if $\phi=-1$, then $\phi=\Delta(1)_{r}^{-}, \mathfrak{a}=(1)_{r}=\mathfrak{l}^{+}(-1)^{2}=\mathfrak{l}^{+}(1)$. The possibilities: $\phi=\Delta(0)_{r}^{-}$and $\Delta(0)_{r}^{+}$cannot arise since $\phi=\Delta(\mathfrak{a})^{+}$or $\Delta(\mathfrak{a})^{-}$for $\mathfrak{a} \neq(0)_{r}$, by hypothesis.

(b) $\Delta(\mathfrak{a})^{2}$ consists of all $t=1+e x(1-e)$ for $e$ such that $(e)_{r}=\mathfrak{a}$, and $\mathfrak{l}^{+}(t)$ $=(e x(1-e))_{\mathfrak{l}}^{r}$ for any $t \in \Delta(\mathfrak{a})^{2}, \quad x \in \Re$. Hence $\mathfrak{a}=\mathfrak{l}^{+} \Delta(\mathfrak{a})^{2}=\bigcap_{t \in \Delta(\mathfrak{a})^{2}} \mathfrak{l}^{+}(t)$ $=\bigcap_{(e) r=a}\left(e x(1-e)_{l}^{r}\right.$.

\section{Multiplicative characterization of $\Delta$-systems.}

THEOREM 2. The set $\phi$ of involutions is a $\Delta$-set if and only if it is a maximal set of involutions satisfying (a) to (d) of Proposition 7.

Proof. If $\phi=\Delta(\mathfrak{a})+$ for some subspace $\mathfrak{a}$, then $\phi$ has properties (a) to (d) of Proposition 7. Suppose $\phi^{\prime}$ is a set of involutions such that $\phi^{\prime} \supset \phi$, and $\phi^{\prime}$ also satisfies (a) to (d). Let $\mathfrak{a}^{\prime}=\mathfrak{l}^{+}\left(\phi^{\prime}\right)^{2}$. Then $\mathfrak{l}^{+}\left(\phi^{\prime}\right)^{2}=\mathfrak{a}^{\prime} \subseteq \mathfrak{a}=\mathfrak{l}^{+}(\phi)^{2}$. Since $\phi^{\prime} \supset \phi$, $\phi^{\prime}$ contains at least two involutions. By Proposition 8, either $\phi^{\prime} \subseteq \Delta\left(\mathfrak{a}^{\prime}\right)+$ or $\phi^{\prime} \subseteq \Delta\left(\mathfrak{a}^{\prime}\right)^{-}$. If $\phi^{\prime} \subseteq \Delta\left(\mathfrak{a}^{\prime}\right)^{-}$, then for every $u \in \phi, \mathfrak{l}-(u)=\mathfrak{a}^{\prime} \subseteq \mathfrak{a}=\mathfrak{l}^{+}(u)$. By 
Lemma $2, \mathfrak{l}^{-}(u)=(0)_{r}, u=1$. But then, by (b), 1 is the only involution in $\phi^{\prime}$, in contradiction to the previous assertion that $\phi^{\prime}$ contains at least two elements. On the other hand, the assumption $\phi^{\prime} \subseteq \Delta(\mathfrak{a})+$ is obviously impossible. It follows that $\phi$ is a maximal set of involutions satisfying (a) to (d).

Conversely, suppose $\phi$ is a maximal set of involutions satisfying (a) to (d). If $\phi$ consists of a single involution $v$, then either $v=1$ or $v=-1$. For, suppose $u$ belongs to $\phi$. Then $\mathfrak{l}^{+}(u)$ and $\mathfrak{l}^{-}(u)$ are complements and, if $\mathfrak{l}^{+}(u) \neq(0)_{r}$, $\neq(1)_{r}$, then $\mathfrak{l}^{+}(u)$ has another complement $\mathfrak{b}$, since the lattice $\mathfrak{l}$ is irreducible. By Lemma 2, there exists an involution $v \neq u$ such that $\mathfrak{l}^{+}(u)=\mathfrak{l}^{+}(v)$. But then the $\Delta$-set $\Delta\left[\mathfrak{l}^{+}(u)\right]^{+}$properly contains $\phi$, contrary to hypothesis. Hence $u=1$ or $u=-1$, and $\phi=\Delta(1)_{r}^{+}$or $\phi=\Delta(1)_{r}^{-}$.

If $\phi$ contains more than one involution, then Proposition 8(3) gives $\phi=\Delta(\mathfrak{a})^{+}$or $\phi=\Delta(\mathfrak{a})^{-}$for $\mathfrak{a}=\mathfrak{l}^{+}\left(\phi^{2}\right)$. This completes the proof.

The $\Delta$-sets are thus completely characterized within the group (s. It follows immediately that the $\Delta$-systems $\left[\Delta(\mathfrak{a})^{+}, \Delta(\mathfrak{a})^{-}\right]=\left[\Delta(\mathfrak{a})^{+},-\Delta(\mathfrak{a})^{+}\right]$are completely characterized within (S).

13. $\Delta$-sets and the partial ordering of $\mathfrak{R}$.

Lemma 19. If $\mathfrak{a}$ and $\mathfrak{b}$ are two subspaces which are not comparable in $\mathfrak{R}$, then there exists a complement $\overline{\mathfrak{a}}$ of $\mathfrak{a}$ such that $(\mathfrak{b} \cap \overline{\mathfrak{a}}) \cup(\mathfrak{b} \cap \mathfrak{a}) \neq \mathfrak{b}$.

Proof. Let $\alpha=\operatorname{dim} \mathfrak{a}, \beta=\operatorname{dim} \mathfrak{b}$. If $\alpha \geqq \beta$, then there exists a subspace $\mathfrak{b}^{\prime}$ such that $\mathfrak{b}^{\prime} \supseteq \mathfrak{b}$ and $\operatorname{dim} \mathfrak{b}^{\prime}=\alpha$. If $\overline{\mathfrak{a}}$ is a common complement of $\mathfrak{a}$ and $\mathfrak{b}^{\prime}$, then $(\mathfrak{b} \cap \overline{\mathfrak{a}}) \cup(\mathfrak{b} \cap \mathfrak{a})=\mathfrak{b} \cap \mathfrak{a} \neq \mathfrak{b}$. If $\alpha<\beta$, let $\mathfrak{a} \cap \mathfrak{b}=\mathfrak{c}$; let $\mathfrak{a}_{1}$ be a relative complement of $c$ in $\mathfrak{a}$, and $\mathfrak{b}_{1}$ a relative complement of $c$ in $\mathfrak{b}_{1}$. If $\operatorname{dim} \mathfrak{c}=\gamma, \operatorname{dim} \mathfrak{a}_{1}=\alpha_{1}$, $\operatorname{dim} \mathfrak{b}_{1}=\beta_{1}$, then $\alpha_{1}=\alpha_{1}-\gamma<\beta-\gamma=\beta_{1}$. Hence $\mathfrak{b}_{1}$ contains a subspace $\mathfrak{b}_{2}$ such that $\operatorname{dim} \mathfrak{b}_{2}=\operatorname{dim} \mathfrak{a}_{1}=\alpha_{1}$. Choose $\mathfrak{b}_{3}$ a relative complement of $\mathfrak{b}_{2}$ in $\mathfrak{b}_{1} ; \mathfrak{g}_{1}$ a common relative complement of $\mathfrak{a}_{1}$ and $\mathfrak{b}_{2}$ in $\mathfrak{a}_{1} \cup \mathfrak{b}_{2}$, and $\mathfrak{g}_{2}$ a complement of $\mathfrak{a} \cup \mathfrak{b}$ in $(1)_{r}$. Then $\overline{\mathfrak{a}}=\mathfrak{b}_{3} \cup \mathfrak{g}_{1} \cup \mathfrak{g}_{2}$ can be shown to have the required properties.

Proposition 9. The following properties of two subspaces $\mathfrak{a}$ and $\mathfrak{b}$ are equivalent.

$$
\begin{aligned}
& \text { I. (a) } \mathfrak{a} \subseteq \mathfrak{b} \text { or }(b) \mathfrak{b} \subseteq \mathfrak{a} \text {, }
\end{aligned}
$$



Proof. Suppose $\mathfrak{a} \subseteq \mathfrak{b}$, and let $u$ be an arbitrary involution in $\Delta(\mathfrak{a})^{+}$, so that $\mathfrak{a}=\mathfrak{l}^{+}(u)$. Then $\mathfrak{b}=\mathfrak{b} \cap(1)_{r}=\mathfrak{b} \cap[\mathfrak{l}-(u) \cup \mathfrak{a}]=\left[\mathfrak{b} \cap \mathfrak{l}^{-}(u)\right] \cup \mathfrak{a}$ by the modular law. If $\mathfrak{c}$ is a relative complement of $\mathfrak{b} \cap \mathfrak{l}^{-}(u)$ in $\mathfrak{I}^{-}(u)$, then $(1)_{r}=\mathfrak{a} \cup \mathfrak{I}^{-}(u)$ $=\mathfrak{a} \cup[(\mathfrak{b} \cap \mathfrak{l}-(u)) \cup \mathfrak{c}]=\left[\mathfrak{a} \cup\left(\mathfrak{b} \cup\left(\mathfrak{b} \cap \mathfrak{l}^{-}(u)\right)\right] \cup \mathfrak{c}=\mathfrak{b} \cup \mathfrak{c}\right.$, and $\mathfrak{b} \cap \mathfrak{c}=(0)_{r}$. Hence there exists a unique involution $v$ such that $\mathfrak{b}=\mathfrak{l}^{+}(v), \mathfrak{c}=\mathfrak{l}^{-}(v)$. From $\mathfrak{l}^{+}(u)$ $=\mathfrak{a} \subseteq \mathfrak{b}=\mathfrak{l}^{+}(v)$ follows $\boldsymbol{\gamma}_{\mathfrak{o}} \mathfrak{l}^{+}(u)=\mathfrak{l}^{+}(u)$. From $\mathfrak{l}^{-}(u)=\left[\mathfrak{b} \cap \mathfrak{l}^{-}(u)\right] \cup \mathfrak{c}=\left[\mathfrak{l}^{+}(v)\right.$ $\left.\cap \mathfrak{I}^{-}(u)\right] \cup_{v}\left[\mathfrak{I}^{-}(v) \cap \mathfrak{I}^{-}(u)\right]$ follows $\gamma_{v} \mathfrak{I}^{-}(u)=\mathfrak{I}^{-}(u)$ (cf. Lemma 14). But then, 
by Proposition 1, $v u=u v$. Since $v$ belongs to $\Delta(\mathfrak{b})^{+}$, it follows by Proposition 7 (c) that $w$ belongs to $N \Delta(\mathfrak{b})^{+}$. But then $\Delta(\mathfrak{a})^{+} \subseteq N \Delta(\mathfrak{b})^{+}$, and we have proved that (a) of I implies II.

Again, suppose $\mathfrak{a} \subseteq \mathfrak{b}$. If $w$ is an arbitrary involution in $\Delta(\mathfrak{b})^{+}$then $\mathfrak{b}=\mathfrak{l}^{+}(w)$. Let $\mathfrak{g}$ be a relative complement of $\mathfrak{a}$ in $\mathfrak{b}$. Then $\mathfrak{a} \cup[\mathfrak{g} \cup \mathfrak{l}-(w)]=(\mathfrak{a} \cup \mathfrak{g}) \cup \mathfrak{l}-(w)$ $=\mathfrak{b} \cup \mathfrak{I}^{-}(w)=\mathfrak{l}^{+}(w) \cup \mathfrak{l}^{-}(w)=(1)_{r}$. Since $(\mathfrak{a} \cup) \mathfrak{g} \cap \mathfrak{I}^{-}(w)=(0)_{r}$, the subspaces $\mathfrak{a}$, $\mathfrak{g}$ and $\mathfrak{I}-(w)$ are independent, and $\mathfrak{a} \cap[\mathfrak{g} \cup \mathfrak{I}-(w)]=(0)_{r}$. Hence there exists a unique involution $s$ such that $\mathfrak{a}=\mathfrak{l}^{+}(s)$ and $\mathfrak{g} \cup \mathfrak{l}-(w)=\mathfrak{l}^{-}(s)$. From $\mathfrak{l}^{+}(s)$ $\subseteq \mathfrak{a} \subseteq \mathfrak{b}=\mathfrak{l}^{+}(w)$ follows $\boldsymbol{\gamma}_{\mathfrak{w}} \mathfrak{l}^{+}(s)=\mathfrak{l}^{+}(s)$, and from $\mathfrak{l}^{-}(s)=\mathfrak{g} \cup \mathfrak{l}^{-}(w)=\left[\mathfrak{l}^{+}(s)\right.$ $\left.\cap \mathfrak{l}^{+}(u)\right] \cup\left[\mathfrak{l}^{-}(s) \cap \mathfrak{l}^{-}(w)\right]$ follows $\gamma_{w} \mathfrak{l}^{-}(s)=\mathfrak{l}^{-}(s)$ (Lemma 14). By Proposition 1 , ws $=s w$, and $w$ belongs to $N\left(\Delta(\mathfrak{a})^{+}\right)$, by Proposition $7(\mathrm{c})$. Hence $\Delta(\mathfrak{b})^{+}$ $\subseteq N \Delta(\mathfrak{a})^{+}$, and we have shown that (a) of I implies both II and III. To show that (b) of I implies both III and II, we need only interchange $\mathfrak{a}$ and $\mathfrak{b}$.

Denying $I$, suppose next that $\mathfrak{a} \nsubseteq \mathfrak{b}$ and $\mathfrak{b} \Phi \mathfrak{a}$. By Lemma 19 , there exists a complement $\overline{\mathfrak{a}}$ of $\mathfrak{a}$ such that $\mathfrak{b} \neq(\mathfrak{b} \cap \mathfrak{a}) \cup(\mathfrak{b} \cap \overline{\mathfrak{a}})$. Let $u$ be the unique involution for which $\mathfrak{a}=\mathfrak{l}^{+}(u), \overline{\mathfrak{a}}=\mathfrak{l}^{-}(u)$, and let $v$ be an arbitrary element of $\Delta(\mathfrak{b})^{+}$. Then $\mathfrak{b}=\mathfrak{l}^{+}(v)$. By Lemma $14, \gamma_{\mathfrak{u}} \mathfrak{b} \neq \mathfrak{b}$. Hence, by Proposition $1, u v=v u$ for $v$ arbitrary in $\Delta(\mathfrak{b})^{+}$and, by Proposition $7(\mathrm{c}), u$ does not belong to $N \Delta(\mathfrak{b})^{+}$. Since $u$ belongs to $\Delta(\mathfrak{a})^{+}$, we conclude that $\Delta(\mathfrak{a})^{+} \subseteq N \Delta(\mathfrak{b})^{+}$. By symmetry of the hypothesis: $\mathfrak{a} \Phi \mathfrak{b}, \mathfrak{b} \Phi \mathfrak{a}$, we have also $\Delta(\mathfrak{b})^{+} \Phi N \Delta(\mathfrak{a})^{+}$. Hence II implies I, and III implies I. The equivalence of I, II, and III is thus established.

\section{Multiplicative characterization of "betweenness."}

Lemma 20. Let $\Delta(\mathfrak{a})^{2}$ denote the subgroup $\left[\Delta(\mathfrak{a})^{+}\right]^{2}=\left[\Delta(\mathfrak{a})^{-}\right]^{2}$. If $t$ is an arbitrary element of the unit group $(S)$ of $\Re$, then $t$ belongs to $\Delta(\mathfrak{a})^{2}$ if and only if $(t-1)_{r} \subseteq \mathfrak{a} \subseteq \mathfrak{l}^{+}(t)=(t-1)_{l}^{r}$.

Proof. If $t$ belongs to $\Delta(\mathfrak{a})^{2}$, then $t-1=e x(1-e)$ for some $e=e^{2} \in \Re$ such that $(e)_{r}=a$ (Proposition $7(\mathrm{~d})$, Lemma 15). But then $(t-1)_{r}=(e x(1-e))_{r}$ $\subseteq(e)_{r}=\mathfrak{a} \subseteq[\operatorname{ex}(1-e)]_{l}^{r}=\mathfrak{l}^{+}(t)=(t-1)_{l}^{r}$.

To prove the converse, suppose $(t-1)_{r} \subseteq \mathfrak{a} \subseteq \mathfrak{l}^{+}(t)$. Then $(t-1)^{2}=0$ since $(t-1)_{r} \subseteq \mathfrak{l}^{+}(t)$. Take $\mathfrak{b}$ a relative complement of $\mathfrak{a}$ in $\mathfrak{l}^{+}(t), \mathfrak{c}$ a complement of $\mathfrak{l}^{+}(t)$ in $(1)_{r}$. Then $\mathfrak{a}, \mathfrak{b}, \mathfrak{c}$ are independent subspaces, and $\mathfrak{c} \cup \mathfrak{b}$ is a complement of $\mathfrak{l}^{+}(t)$. Hence there exists a unique involution $u$ such that $\mathfrak{a}=\mathfrak{l}^{+}(u)$ and $\mathfrak{b} \cup \mathfrak{c}=\mathfrak{l}^{-}(u)$. Clearly, $u$ belongs to $\Delta(\mathfrak{a})^{+}$. Let $v=u t$. Then $v^{2}=1$ and $\mathfrak{l}^{+}(t)$ $=\mathfrak{l}^{+}(u)$. For, $v=u t=u(t-1)+u$. From $(t-1)_{r} \subseteq \mathfrak{a}=\mathfrak{l}^{+}(u)$, we obtain $u(t-1)$ $=t-1, \quad v=t-1+u, \quad v^{2}=(t-1)^{2}+(t-1) u+u(t-1)+u^{2}=(t-1) u+t-1+1$ $=(t-1)(u+1)+1$. But $(t-1)_{r} \subseteq \mathfrak{a}=\mathfrak{l}^{+}(u)=(u+1)_{r} \subseteq \mathfrak{l}^{+}(t)=(t-1)^{r}$. Hence $(t-1)(u+1)=0$, and $v^{2}=1$. Since $\mathfrak{a} \subseteq \mathfrak{l}^{+}(t)$ and $\mathfrak{a}=\mathfrak{l}^{+}(u)$, we have $\mathfrak{a} \subseteq \mathfrak{l}^{+}(v)$.

On the other hand, suppose $x \in \mathfrak{l}^{+}(v)$. Then $x=v x=(t-1+u) x=(t-1) x$ $+u x$. From $u(u-1) x=(1-u) x=-(u-1) x$, we obtain $(t-1) x=-(u-1) x$ $\in(t-1)_{r} \cap \mathfrak{l}^{-}(u) \subseteq \mathfrak{a} \cap(\mathfrak{b} \cup \mathfrak{c})=(0)_{r}$. Hence $u x=x$, so that $x \subseteq \mathfrak{l}^{+}(u)$, and we have $\mathfrak{l}^{+}(v)=\mathfrak{a}=\mathfrak{l}^{+}(u)$. But then, since $t=u v$, and $\mathfrak{l}^{+}(u)=\mathfrak{l}^{+}(v)=v, t$ belongs to $\Delta(\mathfrak{a})^{2}$ as required. 
The following two lemmas are easily established:

Lemma 21. Given $\mathfrak{g}_{1} \subseteq \mathfrak{g}_{2}$, and $\operatorname{dim} \mathfrak{g}_{1}=1-\operatorname{dim} \mathfrak{g}_{2}$. Then there exists $n \in \Re$ such that $n^{2}=0$, and $\cdot g_{1}=(n)_{r}, g_{2}=(n)_{l}^{r}$.

Lemma 22. (a) Given a subspace a of dimension $\rho$, and $M$ the set of all subspaces $m$ of a fixed dimension $\sigma \geqq \rho, \sigma \neq 1$, such that $\mathfrak{m} \supseteq \mathfrak{a}$. Then $\bigcap_{\mathfrak{m} \in M} \mathfrak{m}=\mathfrak{a}$.

(b) Given a subspace $\mathfrak{a}$ of dimension $\rho$ and $N$ the set of all subspaces $\mathfrak{n}$ of $a$ fixed dimension $\tau \leqq \rho, \tau \neq 0$. Then $\mathfrak{a}=\bigcup_{\mathfrak{n} \in N} \mathfrak{n}$.

Proposition 10. If $\mathfrak{a}$ and $\mathfrak{b}$ are two subspaces, then

(a) $(0)_{r}=\mathfrak{a} \cap \mathfrak{b}$ or $(1)_{r}=\mathfrak{a} \cup \mathfrak{b}$ if and only if $\Delta(\mathfrak{a})^{2} \cap \Delta(\mathfrak{b})^{2}=\{1\}$.

(b) (0), $\subset \mathfrak{a} \cap \mathfrak{b} \subseteq \mathfrak{c} \subseteq \mathfrak{a} \cup \mathfrak{b} \subset(1)_{r}$ if and only if $\{1\} \subset \Delta(\mathfrak{a})^{2} \cap \Delta(\mathfrak{b})^{2} \subseteq \Delta(\mathfrak{c})^{2}$.

Proof. By Lemma 20, an element $t$ of (B) belongs to $\Delta(\mathfrak{a})^{2} \cap \Delta(\mathfrak{b})^{2}$ if and only if

$$
(t-1)_{r} \subseteq \mathfrak{a} \cap \mathfrak{b} \leqq \mathfrak{a} \cup \mathfrak{b} \subseteq(t-1)_{\mathfrak{l}}^{r} .
$$

(a) If $(0)_{r}=\mathfrak{a} \cap \mathfrak{b}$, or $(1)_{r}=\mathfrak{a} \cup \mathfrak{b}$, then $\Delta(\mathfrak{a})^{2} \cap \Delta(\mathfrak{b})^{2}=\{1\}$ as an immediate consequence of $(C)$. To prove the converse, suppose that $(0)_{r} \subset \mathfrak{a} \cap \mathfrak{b}$ and $\mathfrak{a} \cup \mathfrak{b} \subset(1)_{r}$, and let $\mathfrak{g}_{1}$ and $\mathfrak{g}_{2}$ be subspaces such that $(0)_{r} \subset \mathfrak{g}_{1} \subseteq \mathfrak{a} \cap \mathfrak{b} \subseteq \mathfrak{a} \cup \mathfrak{b}$ $\subseteq g_{2} \subset(1)_{r}$, and $\operatorname{dim} g_{1}+\operatorname{dim} g_{2}=1$. Select $g_{1}$ so that $\operatorname{dim} g_{1} \leq \min [\operatorname{dim}(\mathfrak{a}$ $\widehat{\cap} \mathfrak{b}), 1-\operatorname{dim}(\mathfrak{a} \cup \mathfrak{b})]$, and then select $\mathfrak{g}_{2}$ as indicated.) By Lemma 21 , there exists $n \in \Re$ such that $n^{2}=0$ and $g_{1}=(n)_{r}, g_{2}=(n)_{l}^{r}$. Since $g_{1} \neq(0)_{r}, n$ is different from 0 , and by Lemma $20, t=1+n$ belongs to $\Delta(\mathfrak{a})^{2} \cap \Delta(\mathfrak{b})^{2}$. But $t$ is different from 1 . Hence $\Delta(\mathfrak{a})^{2} \cap \Delta(\mathfrak{b})^{2} \neq\{1\}$, and we have shown that $\Delta(\mathfrak{a})^{2}$ $\cap \Delta(\mathfrak{b})^{2}=\{1\}$ implies $\mathfrak{a} \cap \mathfrak{b}=(0)_{r}$, or $\mathfrak{a} \cup \mathfrak{b}=(1)_{r}$.

(b) The necessity is an immediate consequence of (a) and Lemma 20. To prove the sufficiency, suppose $\Delta(\mathfrak{a})^{2} \cap \Delta(\mathfrak{b})^{2} \subseteq \Delta(\mathfrak{c})^{2}$. For every $n=t-1$ such that $(n)_{r} \subseteq \mathfrak{a} \cap \mathfrak{b} \subseteq \mathfrak{a} \cup \mathfrak{b} \subseteq(n)_{l}^{r}$, we have $(n)_{r} \subseteq \mathfrak{c} \subseteq(n)_{l}^{r}$. If $\operatorname{dim}(\mathfrak{a} \cap \mathfrak{b}) \leqq 1$ $-\operatorname{dim}(\mathfrak{a} \cup \mathfrak{b})$, let $\mathfrak{g}_{1}=\mathfrak{a} \cap \mathfrak{b}$. Then there exist subspaces $\mathfrak{g}$ such that $\operatorname{dim} \mathfrak{g}$ $=1-\operatorname{dim}(\mathfrak{a} \cap \mathfrak{b})$ and $\mathfrak{a} \cup \mathfrak{b} \subseteq \mathfrak{g}$. By Lemma 21 , there exists for each $\mathfrak{g}$ an $n_{\mathfrak{g}}\left(n_{\mathfrak{g}}^{2}=0\right)$ such that $\mathfrak{g}_{1}=\left(n_{\mathfrak{g}}\right)_{r}, \mathfrak{g}=\left(n_{\mathfrak{g}}\right)_{l}^{r}$, and we have $(0)_{r} \subset \mathfrak{g}_{1}=\left(n_{\mathfrak{g}}\right)_{r}$ $\subseteq \mathfrak{a} \cup \mathfrak{b} \subseteq \mathfrak{g}=\left(n_{\mathfrak{g}}\right)_{\mathfrak{l}}^{r}$ for every such $\mathfrak{g}$ and $n_{\mathfrak{g}}$. Therefore $\mathfrak{a} \cap \mathfrak{b}=\left(n_{\mathfrak{g}}\right)_{\mathfrak{r}} \subseteq \mathfrak{c} \subseteq\left(n_{\mathfrak{g}}\right)_{\mathfrak{l}}^{r}$ for every such $n_{\mathfrak{g}}$, and

$$
\mathfrak{a} \cap \mathfrak{b} \subseteq \mathfrak{c} \subseteq \cap\left(n_{\mathfrak{g}}\right)_{l}^{r}=\bigcap_{\mathfrak{g}}
$$

for $\mathfrak{g}$ with $\operatorname{dim} \mathfrak{g}=1-\operatorname{dim}(\mathfrak{a} \cap \mathfrak{b})$ and $\mathfrak{g} \supseteq \mathfrak{a} \cup \mathfrak{b}$. By Lemma 22(a), $\cap \mathfrak{g}=\mathfrak{a} \cup \mathfrak{b}$, and $\mathfrak{a} \cap \mathfrak{b} \subseteq \mathfrak{c} \subseteq \mathfrak{a} \cup \mathfrak{b}$. If $\operatorname{dim}(\mathfrak{a} \cap \mathfrak{b})>1-\operatorname{dim}(\mathfrak{a} \cup \mathfrak{b})$, the dual argument, using Lemma 27 (b), gives once more that $\mathfrak{a} \cap \mathfrak{b} \subseteq \mathfrak{c} \subseteq \mathfrak{a} \cup \mathfrak{b}$.

Theorem 3. Let $\mathfrak{a}, \mathfrak{b}$, and $\mathfrak{c}$ be three subspaces. Then $\mathfrak{a}$ and $\mathfrak{b}$ are both different from $(0)_{r}$ and $(1)_{r}$, and $\mathfrak{c}$ is between $\mathfrak{a}$ and $\mathfrak{b}$ if and only if

(a) $\Delta(\mathfrak{a}) \subseteq N \Delta(\mathfrak{b})$ or $\Delta(\mathfrak{b}) \subseteq N \Delta(\mathfrak{a})$ and

(b) $\{1\} \subset \Delta(\mathfrak{a})^{2} \cap \Delta(\mathfrak{b})^{2} \subseteq \Delta(\mathfrak{c})^{2}$.

This theorem is an immediate consequence of Propositions 9 and 10. 


\section{Correspondence between $\Delta$-systems and subspaces.}

THEOREM 4.

(a) The following properties of a subspace $\mathfrak{a}$ are equivalent:

(1) $\mathfrak{a}=(0)_{\text {r }}$ or $(1)_{r}$,

(2) $\Delta(\mathfrak{a})=[\{1\},\{-1\}]$

(3) $\Delta(\mathfrak{a})^{2}=\{1\}$,

(4) $N \Delta(\mathfrak{a})$ consists of all the involutions in $\Re$.

(b) If $(0)_{r} \subset \mathfrak{a} \subseteq(1)_{r}$, then $\mathfrak{a}=\mathfrak{l}^{+}\left[\Delta(\mathfrak{a})^{2}\right]$.

(c) If $\phi$ is a maximal set of involutions satisfying conditions (a) to (d) of Proposition 7, then $\Delta\left[\mathfrak{l}^{+}\left(\phi^{2}\right)\right]=[\phi,-\phi]$.

Proof. (a) The implications: $1 \Rightarrow 2,2 \Rightarrow 3,3 \Rightarrow 4$ are obvious. By Proposition 9 , we have: $4 \Rightarrow 1$.

(b) This was proved as Corollary 1 .

(c) If $\phi$ is a maximal set of involutions satisfying (a) to (d) of Proposition 7 , containing at least two involutions, then Proposition 8 requires $\Delta\left[\mathfrak{l}^{+}\left(\phi^{2}\right)\right]$ $=[\phi,-\phi]$. If $\phi$ consists of a single involution, then $[\phi,-\phi]=[\{1\},\{-1\}]$, and $\Delta\left[\mathfrak{l}^{+}\left(\phi^{2}\right)\right]=[\{1\},\{-1\}]$ by (a) of Proposition 7 .

TheOREM 5. Mapping a onto $\Delta(\mathfrak{a})=\left[\Delta(\mathfrak{a})^{+}, \Delta(\mathfrak{a})^{-}\right]$, and mapping $[\phi,-\phi]$ upon $\mathfrak{I}^{+}\left(\phi^{2}\right)$ constitute reciprocal (1-1) correspondences between the set of all subspaces, not $(0)_{r}$, and the set of all $\Delta$-systems in $\$$.

Proof. By Theorem 2, the mapping $\mathfrak{a} \rightarrow \Delta(\mathfrak{a})$ is single-valued. By Theorem 4 , every $\Delta$-system has a unique inverse image $a \neq(0)_{r}$, and the mappings $\mathfrak{a} \neq(0)_{r} \rightarrow \Delta(\mathfrak{a})$ and $\left[\phi_{1}-\phi\right] \rightarrow \mathfrak{l}^{+}\left(\phi^{2}\right)$ are reciprocal.

16. Characterization of $\Delta$-systems in terms of the derived systems $\Delta^{2}$.

Proposition 11. Given $\mathfrak{a} \neq(0)_{r}, \neq(1)_{r}$. Then $u$ belongs to $\Delta(\mathfrak{a})$ if and only if $u t u=t^{-1}$ for every $t$ in $\Delta(\mathfrak{a})^{2}$.

Proof. If $u$ is an element of $\Delta(\mathfrak{a})=\left[\Delta(\mathfrak{a})^{+}, \Delta(\mathfrak{a})^{-}\right]$, then $u t u=t^{-1}$ for every $t \in \Delta(\mathfrak{a})^{2}$, by Propositions 7 and 8 . Conversely, suppose $u t u=t^{-1}$ for every $t$ in $\Delta(\mathfrak{a})^{2}$. If $t=1+e x(1-e)$ for $x \in \Re$ and $e=e^{2}$ such that $(e)_{r}=\mathfrak{a}$, then $u t u=t^{-1}$ implies that $u e$ belongs to $(e x(1-e))_{l}^{r}$. By Corollary $1(\mathrm{~b}), \quad(u e)_{r}=\gamma_{u} \mathrm{a}$ $\subseteq \bigcap_{x \in \mathfrak{R},(e),=\mathfrak{a}}(e x(1-e))_{\mathfrak{l}}^{r}=\mathfrak{l}^{+}[\Delta(\mathfrak{a})]^{2}=\mathfrak{a}$. By Lemma 14, $\mathfrak{a}=\mathfrak{a}^{+} \cup \mathfrak{a}^{-}$, where $\mathfrak{a}^{+}=\mathfrak{a} \cap \mathfrak{l}^{+}(u)$ and $\mathfrak{a}^{-}=\mathfrak{a} \cap \mathfrak{l}^{-}(u)$. Let $\mathfrak{b}^{+}$be a relative complement of $\mathfrak{a}^{+}$in $\mathfrak{l}^{+}(u)$, and let $\mathfrak{b}^{-}$be a relative complement of $\mathfrak{a}^{-}$in $\mathfrak{l}^{-}(u)$. Then $\mathfrak{a}^{+}, \mathfrak{b}^{+}, \mathfrak{a}^{-}$, and $\mathfrak{b}^{-}$are independent subspaces [1, Theorem 2.2 , p. 11]. Hence there exist mutually orthogonal idempotents $g_{1}, g_{2}, h_{1}, h_{2}$ such that $\left(g_{1}\right)_{r}=\mathfrak{a}^{+},\left(g_{2}\right)_{r}=\mathfrak{a}^{-}$, $\left(h_{1}\right)_{r}=\mathfrak{b}^{+},\left(h_{2}\right)_{r}=\mathfrak{b}^{-}$, and $g_{1}+g_{2}+h_{1}+h_{2}=1$ (Lemma 11).

From $\mathfrak{l}^{+}(u)=\left(g_{1}\right)_{r} \cup\left(h_{1}\right)_{,}=\left(g_{1}+h_{1}\right)_{r}$ and $\mathfrak{l}^{-}(u)=\left(g_{2}\right)_{r} \cup\left(h_{2}\right)_{r}=\left(g_{2}+h_{2}\right)_{r}$, using Lemma 2, we obtain the relations: (A): $(1+u)=2\left(g_{1}+h_{1}\right)$, and (B): $1-u=2\left(g_{2}+h_{2}\right)$. From $g_{1}, h_{1} \in \mathfrak{l}^{+}(u), g_{2}, h_{2} \in \mathfrak{l}^{-}(u)$, we obtain $u g_{1}=g_{1}$, 
$u h_{1}=h_{1}, u g_{2}=-g_{2}, u h_{2}=-h_{2}$. From (A) and (B) we now have by leftmultiplication: $g_{1}=g_{1} u, h_{1}=h_{1} u, g_{2}=-g_{2} u, h_{2}=-h_{2} u$.

Since $\left(g_{1}\right)_{r} \cup\left(g_{2}\right)_{r}=\left(g_{1}+g_{2}\right)_{r}=\mathfrak{a}, \quad t=1+\left(g_{1}+g_{2}\right) x\left(1-g_{1}-g_{2}\right)=1+\left(g_{1}+g_{2}\right)$ $\cdot x\left(h_{1}+h_{2}\right)$ belongs to $\Delta(\mathfrak{a})^{2}$ for $x$ arbitrary in $\Re$. Hence $u t=t^{-1} u$ or $u+u\left(g_{1}+g_{2}\right)$ $\cdot x\left(h_{1}+h_{2}\right)=u-\left(g_{1}-g_{2}\right) x\left(h_{1}+h_{2}\right) u$, so that $(\mathrm{C}):\left(g_{1}-g_{2}\right) x\left(h_{1}+h_{2}\right)=-\left(g_{1}+g_{2}\right)$ $\cdot x\left(h_{1}-h_{2}\right)$. On left-multiplication of (C) by $g_{1}$, we obtain $g_{1} x h_{1}=0$ for $x$ arbitrary in $\Re$. On right-multiplication of (C) by $g_{2}$, we obtain $g_{2} x h_{2}=0$ for $x$ arbitrary in $\Re$. By Lemma 13 , either $g_{1}=0$ or $h_{1}=0$; and, either $g_{2}=0$ or $h_{2}=0$. Since $\mathfrak{a} \neq(0)_{r}, \mathfrak{a} \neq(1)_{r}$, we have either $g_{1}=0$ and $h_{2}=0$, or $g_{2}=0$ and $h_{1}=0$. In the first case, $\mathfrak{a}=\left(g_{2}\right)_{r}=\mathfrak{I}^{-}(u)$; in the second case, $\mathfrak{a}=\left(g_{1}\right)_{r}=\mathfrak{l}^{+}(u)$. In either case, $u$ belongs to $\Delta(\mathfrak{a})$. This completes the proof.

\section{PART IV. ISOMORPHISM AND STRUCTURE THEOREMS}

17. Conditions on $\Re$. We assume as before that $\Re$ is the continuous ring associated with a continuous geometry $\mathbb{R}$, and that $\Re$ does not have characteristic 2. This latter condition is expressible as a condition on the unit group (5) of $\Re$, since $2=0$ if and only if 1 has a unique square root in $B$.

18. Induced isomorphisms; singular automorphisms. If $\Re_{1}$ and $\Re_{2}$ are continuous rings with unit groups $\mathscr{S}_{1}$ and $\mathfrak{F}_{2}$, and $\alpha$ is an isomorphism of $\Re_{1}$ onto $\Re_{2}$, then clearly $\alpha$ induces an isomorphism of $\mathbb{S}_{1}$ onto $\mathcal{S}_{2}$. If $\beta$ is an antiisomorphism of $\Re_{1}$ onto $\Re_{2}$, then again $\beta$ induces an isomorphism of $\mathscr{S}_{1}$ onto $\mathrm{G}_{2}$, since $\mathrm{B}_{2}$ is anti-automorphic to itself under the mapping $x \rightarrow x^{-1}, x \in \mathrm{G}_{2}$.

DEFINITION 5. An automorphism of the unit group \& of a continuous ring will be called singular if it is of the form $x \rightarrow z_{x} x(x \in \mathfrak{S})$, where $z_{x}$ is an element of the center $\overline{3}$ of $B$ depending on $x$ (cf. [8, p. 230]).

LEMMA 23. The mapping $x \rightarrow z_{x}$ is a homomorphism of $\&$ into its center $\overline{3}$ if and only if the mapping $x \rightarrow z_{x} x$ is a singular automorphism of (5).

The proof is completely straightforward.

We show in the following that every isomorphism of $\mathfrak{S}_{1}$ onto $\mathfrak{S}_{2}$ may be represented uniquely as the product of a singular automorphism of $\oiint_{1}$ by an induced isomorphism of $\mathrm{S}_{1}$ onto $\mathrm{BH}_{2}$, (Isomorphism Theorem). From this, we deduce that the structure of a continuous ring, and hence the structure of the associated geometry, is completely determined within the unit group \&, (Structure Theorem).

Proposition 12. The following properties of the automorphism $\sigma$ of \&s are equivalent:

I. $\sigma$ is singular.

II. $\sigma$ leaves invariant every element of class 2 .

III. $\Delta(\mathfrak{a})^{\sigma}=\Delta(\mathfrak{a})$ for every subspace $\mathfrak{a}$.

The proof is completely analogous to Baer's proof for the projective case $[8$, p. 231]. 
Lemma 24. For any subspace $a \neq(0)_{r}, \not(1)_{r}$,

(a) $\Delta(\mathfrak{a})^{2}-1=a^{l} \cap \mathfrak{a}$,

(b) $\left(\mathfrak{a}^{l} \cap \mathfrak{a}\right)^{r}=\mathfrak{a}$,

(c) $\left(\mathfrak{a}^{l} \cap \mathfrak{a}\right)^{l}=\mathfrak{a}^{l}$,

where $\Delta(\mathfrak{a})^{2}-1$ denotes the set of all $t-1$ for which $t \in \Delta(\mathfrak{a})^{2}$, and right and left annihilators are indicated by superscripts $r$ and $l$, respectively [5, p. 709].

Proof. Setting $\mathfrak{a}=(e)$, for $e=e^{2} \in \Re$, and observing that $\Delta(\mathfrak{a})^{2}-1$ is the set of all $e x(1-e)$ for $x \in \Re$, one readily verifies (a), (b) and (c).

Proposition 13. The identity is the only automorphism of (B) which is at the same time singular and induced.

Proof. Let $\sigma$ be a singular automorphism of $\$$. By Proposition $12, \Delta(\mathfrak{a})^{2}$ $=\left[\Delta(\mathfrak{a})^{2}\right]^{\sigma}$ for every subspace $\mathfrak{a}$. Suppose $\alpha$ is an automorphism of $\Re$, inducing $\sigma$ on $\mathbb{B}$, and $\mathfrak{a}=(e)_{r}$ is a subspace $\neq(0)_{r}, \neq(1)_{r}$. By Lemma $24,\left[\mathfrak{a}^{l} \cap \mathfrak{a}\right]^{\alpha}$ $=\left[\Delta(\mathfrak{a})^{2}-1\right]^{\alpha}=\left[\Delta(\mathfrak{a})^{2}\right]^{\alpha}-1$. Since $\Delta(\mathfrak{a})^{2}$ is a subgroup of $\mathfrak{B},\left[\Delta(\mathfrak{a})^{2}\right]^{\alpha}-1$ $=\left[\Delta(\mathfrak{a})^{2}\right]^{\sigma}-1=\mathfrak{a}^{l} \cap \mathfrak{a}$, so that $\left[\mathfrak{a}^{l} \cap \mathfrak{a}\right]^{\alpha}=\mathfrak{a}^{l} \cap \mathfrak{a}$. Hence $\mathfrak{a}^{\alpha}=\left[\left(\mathfrak{a}^{l} \cap \mathfrak{a}\right)^{r}\right]^{\alpha}$ $=\left[\left(\mathfrak{a}^{l} \cap \mathfrak{a}\right)^{\alpha}\right]^{r}=\left(\mathfrak{a}^{l} \cap \mathfrak{a}\right)^{r}=\mathfrak{a}$. Clearly, $0^{\alpha}=0,1^{\alpha}=1$, so that $(0)_{r}^{\alpha}=(0)_{r}$ and $(1)_{r}^{\alpha}=(1)_{r}$. But then the ring automorphism $\alpha$ induces the identity automorphism on the lattice $\mathfrak{R}$. Since the correspondence between ring automorphisms and lattice automorphisms is 1-1 [2, pp. 42-48], $\alpha$ is the identity on $\Re$, and hence the singular automorphism $\sigma$ is the identity on $\mathbb{S}$.

Suppose next that $\beta$ is an anti-automorphism of $\Re$, inducing $\sigma$ on $B$, so that $t^{\sigma}=\left(t^{\beta}\right)^{-1}$ for $t \in(B)$. Using Lemma 24, and noting that an anti-automorphism of $\Re$ interchanges right and left annihilators, and maps right ideals to left ideals $\left[5\right.$, Theorem 1, p. 709] we have $\left[\mathfrak{a}^{l} \cap \mathfrak{a}\right]^{\beta}=\left[\Delta(\mathfrak{a})^{2}-1\right]^{\beta}=\left[\Delta(\mathfrak{a})^{2}\right]^{\beta}$ $-1^{\beta}=\left\{\left[\Delta(\mathfrak{a})^{2}\right]^{\sigma}\right\}^{-1}-1=\left[\Delta(\mathfrak{a})^{2}\right]^{-1}-1=\Delta(\mathfrak{a})^{2}-1=\mathfrak{a}^{2} \cap \mathfrak{a}$. But then $(\mathfrak{a})^{\beta}$ $=\left[\left(\mathfrak{a}^{l} \cap \mathfrak{a}\right)^{r}\right]^{\beta}=\left[\left(\mathfrak{a}^{l} \cap \mathfrak{a}\right)^{\beta}\right]^{l}=\left(\mathfrak{a}^{l} \cap \mathfrak{a}\right)^{l}=\mathfrak{a}$ for all $\mathfrak{a} \neq(0)_{r}, \neq(1)_{r}$. Hence the antiautomorphism $\left({ }^{2}\right)$ of the lattice $\mathfrak{R}$ induced by $\beta$ (cf. [2, Theorem 4.3, p. 48]) coincides with the identity on all subspaces $\neq(0)_{r}, \neq(1)_{r}$. This is impossible, as partial order must be reversed. Thus, an anti-automorphism of $\Re$ cannot induce a singular automorphism on $(\mathcal{G}$, and we have shown that the only singular automorphism which can be induced by a ring automorphism is the identity.

19. Betweenness and partial order.

Lemмa 25. If $\sigma$ is a 1-1 mapping of the totality of proper subspaces (i.e., subspaces $\left.\neq(0)_{r}, \neq(1)_{r}\right)$ of a continuous geometry $\mathfrak{R}_{1}$ onto the totality of proper subspaces of a continuous geometry $\mathfrak{R}_{2}$, preserving betweenness, then $\sigma$ is induced by an isomorphism or by an anti-isomorphism of $\mathfrak{R}_{1}$ onto $\mathfrak{R}_{2}$.

Proof. If $\mathfrak{a}$ is a proper subspace $\in \mathfrak{R}_{1}$, let $\mathfrak{a}$ be called "order-preserving" if $\mathfrak{l} \supseteq \mathfrak{a}$ implies $\sigma(\mathfrak{a}) \subseteq \sigma(\mathfrak{l})$, and $\mathfrak{l} \subset \mathfrak{a}$ implies $\sigma(\mathfrak{l}) \subset \sigma(\mathfrak{a})$. Then $\mathfrak{a}$ is order-preserv-

(2) Here, we use the term "anti-automorphism" in the sense of "dual automorphism." 
ing if $\sigma(\mathfrak{b}) \supseteq \sigma(\mathfrak{a})$ for a single $\mathfrak{b} \supseteq \mathfrak{a}$, or $\sigma(b) \subset \sigma(\mathfrak{a})$ for a single $\mathfrak{b} \subset \mathfrak{a}$. For if $\mathfrak{b} \subset \mathfrak{a}$ and $\mathfrak{t} \subset \mathfrak{a}$, then $\mathfrak{a}$ is not between $\mathfrak{b}$ and $\mathfrak{l}$, and hence $\sigma(\mathfrak{a})$ is not between $\sigma(\mathfrak{b})$ and $\sigma(\mathfrak{l})$. Since $\sigma(\mathfrak{b}) \subset \sigma(\mathfrak{a})$, it follows that $\sigma(\mathfrak{a}) \subset \sigma(\mathfrak{l})$ is impossible. But the only alternative, since betweenness is preserved, is $\sigma(\mathfrak{l}) \subset \sigma(\mathfrak{a})$, as required. Similar arguments apply in the other cases.

Thus if $\mathfrak{a}$ is order-preserving, and $(\mathfrak{a}, \mathfrak{m}),(\mathfrak{m}, \mathfrak{n})$ are pairs of comparable subspaces, then $n$ is also order-preserving. Now, if $\mathfrak{a} \subset \mathfrak{b}$ holds for a single pair with $\sigma(\mathfrak{a}) \subset \sigma(\mathfrak{b})$, let $\mathfrak{b}$ be arbitrary. It follows that $(0)_{r} \subset(\mathfrak{b} \cap \mathfrak{b}) \subset(1)_{r}$, or $(0)_{r} \subset \mathfrak{a} \cup \mathfrak{b} \subset(1)_{r}$. For, if $\mathfrak{a} \cup \mathfrak{b}=(1)_{r}$, then $\mathfrak{b}=\mathfrak{b} \cap(\mathfrak{a} \cup \mathfrak{b})=\mathfrak{a} \cup(\mathfrak{b} \cap \mathfrak{b})$, so that $\mathfrak{b} \cap \mathfrak{b} \neq(0)_{r}$. If $\mathfrak{a} \cup \mathfrak{d} \subset(1)_{r}$, then $\mathfrak{a} \subseteq \mathfrak{a} \cup \mathfrak{b}, \mathfrak{b} \subseteq \mathfrak{a} \cup \mathfrak{b}$; if $\mathfrak{b} \cap \mathfrak{b} \neq(0)_{r}$, then $\mathfrak{b} \supseteq \mathfrak{b} \cap \mathfrak{d}, \mathfrak{b} \supseteq \mathfrak{b} \cap \mathfrak{d}$, so that in every case $\mathfrak{b}$ is order-preserving; thus $\mathfrak{c} \subseteq \mathfrak{d}$ implies $\sigma(\mathfrak{c}) \subseteq \sigma(\mathfrak{b})$ for all $\mathfrak{c}, \mathfrak{b} \in \mathfrak{R}_{1}$. But then, if $\mathfrak{a} \subset \mathfrak{b}$ holds for a single pair with $\sigma(\mathfrak{a}) \supseteq \sigma(\mathfrak{b}), \mathfrak{c} \subseteq \mathcal{b}$ implies $\sigma(\mathfrak{c}) \supseteq \sigma(\mathfrak{b})$ for all $\mathfrak{c}, \mathfrak{b} \in \mathfrak{R}_{1}$.

\section{Isomorphism theorem.}

THEOREM 6. Let $\mathfrak{B}_{1}$ and $\mathfrak{H}_{2}$ be the unit groups of two continuous rings $\Re_{1}$ and $\Re_{2}(2 \neq 0)$. Then any isomorphism $\sigma$ of $\mathfrak{S}_{1}$ onto $\mathfrak{S}_{2}$ may be uniquely represented in the form $\sigma=\sigma^{\prime \prime} \sigma^{\prime}$ where $\sigma^{\prime \prime}$ is a singular automorphism, and $\sigma^{\prime}$ is an induced isomorphism.

Proof. Let $\Re_{1}$ and $\Re_{2}$ have corresponding geometries $\mathfrak{R}_{1}$ and $\mathfrak{R}_{2}$. If $\mathfrak{a}$ is any proper subspace of $\Re_{1}$, and $\sigma$ is an isomorphism of $\mathfrak{S}_{1}$ onto $\mathfrak{G}_{2}$, then, by Theorem 2, $[\Delta(\mathfrak{a})] \sigma$ is again a $\Delta$-system, with elements in $\mathfrak{S}_{2}$. By Theorem $4, \sigma$ induces a 1-1 mapping $\mu: \mathfrak{a} \rightarrow \mathfrak{a}^{u}=\mathfrak{I}^{+}\left\{[\Delta(\mathfrak{a})]^{\sigma}\right\}^{2}$ of the totality of proper subspaces of $\mathfrak{R}_{1}$ onto the totality of proper subspaces of $\mathfrak{R}_{2}$. By Theorem 3 , the mapping $\mu$ preserves betweenness and, according to Lemma 25 , is therefore induced by an isomorphism or an anti-isomorphism of the lattice $\ell_{1}$ onto the lattice $\mathfrak{R}_{2}$. Let $\mu$ denote this mapping also and suppose first that $\mu$ is a lattice isomorphism. Then there exists a unique isomorphism of $\Re_{1}$ onto $\Re_{2}$ inducing $\mu$. From Lemma 24 and the fact that $\sigma^{\prime}$ preserves the annihilator relation it follows that, for every proper subspace $\mathfrak{a},\left[\Delta(\mathfrak{a})^{2}\right]^{\sigma^{\prime}}-1=\left[\Delta(\mathfrak{a})^{2}-1\right]^{\sigma^{\prime}}=\left(\mathfrak{a}^{l}\right.$ $\cap \mathfrak{a})^{\sigma^{\prime}}=\left(\mathfrak{a}^{l}\right)^{\sigma^{\prime}} \cap \mathfrak{a}^{\sigma^{\prime}}=\left(\mathfrak{a}^{\sigma^{\prime}}\right)^{l} \cap \mathfrak{a}^{\sigma^{\prime}}=\left(\mathfrak{a}^{\mu}\right)^{l} \cap \mathfrak{a}^{\mu}=\Delta\left(\mathfrak{a}^{\mu}\right)^{2}-1$, so that $\left[\Delta(\mathfrak{a})^{2}\right]^{\sigma^{\prime}}$ $=\Delta(\mathfrak{a})^{2}$. Since $\sigma^{\prime}$ induces an isomorphism $\left(\sigma^{\prime}\right)$ of $\mathfrak{S}_{1}$ onto $\mathfrak{H}_{2},\left[\Delta(\mathfrak{a})^{2}\right]^{\sigma^{\prime}}$ $=\left[\Delta(\mathfrak{a})^{\sigma^{\prime}}\right]^{2}$, so that $\left[\Delta(\mathfrak{a})^{\sigma^{\prime}}\right]^{2}=\left[\Delta(\mathfrak{a})^{\mu}\right]^{2}$. Hence, by Theorem 4 (or by Proposition 11), $\Delta(\mathfrak{a})^{\sigma^{\prime}}=\Delta\left(\mathfrak{a}^{\mu}\right)=[\Delta(\mathfrak{a})]^{\sigma}$ for all proper subspaces $\mathfrak{a}$. If $\mathfrak{a}=(0)_{r}$ or $\mathfrak{a}=(1)_{r}, \Delta(\mathfrak{a})=[+1,-1]$, (Theorem 4), and $[\Delta(\mathfrak{a})]^{\sigma^{\prime}}=[+1,-1]=\Delta(\mathfrak{a})^{\sigma}$. But then $[\Delta(\mathfrak{a})]^{\sigma\left(\sigma^{\prime}\right)}-1=\Delta(\mathfrak{a}) \in \mathfrak{S}_{1}$ for every $\mathfrak{a} \in \mathfrak{R}_{1}$. Hence, by Proposition 12, $\sigma^{\prime \prime} \equiv \sigma\left(\sigma^{\prime}\right)^{-1}$ is a singular automorphism of $\oiint_{1}$ and $\sigma=\sigma^{\prime \prime} \sigma^{\prime}$ is a representation of $\sigma$ in the required form. The uniqueness follows by Proposition 13.

Suppose next that $\mu$ is an anti-isomorphism of $\mathfrak{l}_{1}$ onto $\mathfrak{R}_{2}$. Then there exists a unique anti-isomorphism $\sigma^{\prime}$ of $\Re_{1}$ onto $\Re_{2}$ such that $(a)_{r}^{\mu}=\left(a^{\sigma^{\prime}}\right)_{l}^{r}$ for every subspace $a=(a)_{r}$ of $\mathfrak{R}_{1}\left[2\right.$, p. 48]. If $\mathfrak{a}=(e)$, is a proper subspace of $\mathfrak{R}_{1}$ and $e=e^{2} \in \Re$, then $\left[\Delta(\mathfrak{a})^{2}\right]^{\sigma^{\prime}}-1=\left[\Delta(\mathfrak{a})^{2}-1\right]^{\sigma^{\prime}}=\left[\mathfrak{a} \cap \mathfrak{a}^{l}\right] \sigma^{\sigma^{\prime}}=\left[\mathfrak{a}^{\sigma^{\prime}} \cap \mathfrak{a}^{l}\right]^{\sigma^{\prime}}=\left(e^{\sigma^{\prime}}\right)_{l}$ $\cap\left(1-e^{\sigma^{\prime}}\right)_{r}=\left(1-e^{\sigma^{\prime}}\right)_{r}^{l} \cap\left(e^{\sigma^{\prime}}\right)_{l}^{r}=\left(\mathfrak{a}^{\mu}\right)^{l} \cap \mathfrak{a}^{\mu}=\Delta\left(\mathfrak{a}^{\mu}\right)^{2}-1$. Hence, as before, 
$\left[\Delta(\mathfrak{a})^{2}\right]^{\sigma^{\prime}}=\Delta\left(\mathfrak{a}^{\mu}\right)^{2}$ for every proper subspace $\mathfrak{a}$.

If $-\sigma^{\prime}$ denotes the isomorphism of $\mathfrak{S}_{1}$ onto $\mathfrak{B H}_{2}$ induced by $\sigma^{\prime}$, then $\left[\Delta(\mathfrak{a})^{2}\right] \sigma^{\prime}$ $=\left[\Delta(\mathfrak{a})^{2}\right]^{-\sigma^{\prime}}$ since $\Delta(\mathfrak{a})^{2}$ is a group; $\left[\Delta(\mathfrak{a})^{2}\right]^{-\sigma^{\prime}}=\left[\Delta(\mathfrak{a})^{-\sigma^{\prime}}\right]^{2}$ since $-\sigma^{\prime}$ is an isomorphism; and $\Delta(\mathfrak{a})^{-\sigma^{\prime}}=\Delta(\mathfrak{a})^{\sigma^{\prime}}$ since $u^{-1}=u$ for every $u \in \Delta(\mathfrak{a})$. Hence, as before, $\Delta(\mathfrak{a})^{\sigma^{\prime}}=\Delta\left(\mathfrak{a}^{\mu}\right)=\Delta(\mathfrak{a})^{\sigma}$ for all proper subspaces $\mathfrak{a}$. If $\mathfrak{a}=(0)_{r}$ or $\mathfrak{a}=(1)_{r}$, then $\Delta(\mathfrak{a})^{\sigma^{\prime}}=[1,-1]=\Delta(\mathfrak{a})$. Thus $[\Delta(\mathfrak{a})]^{\sigma\left(\sigma^{\prime}\right)^{-1}}=\Delta(\mathfrak{a})$ for every $\mathfrak{a} \in \mathfrak{R}_{1}$. By Proposition $12, \sigma^{\prime \prime}=\sigma\left(\sigma^{\prime}\right)^{-1}$ is a singular automorphism of $\mathfrak{H}_{1}$, and $\sigma=\sigma^{\prime \prime} \sigma^{\prime}$ is a representation of $\sigma$ in the required form. Since the product of two antiisomorphisms is a direct isomorphism, the uniqueness follows as in the previous case.

\section{Structure theorem.}

THEOREM 7. Suppose $2 \neq 0$. (a) Two continuous rings are isomorphic or anti-isomorphic if and only if their unit groups are isomorphic. (b) Equivalently, two continuous geometries are isomorphic or anti-isomorphic if and only if the unit groups of the associated continuous rings are isomorphic.

Proof. (a) This is an immediate consequence of Theorem 6. (b) The equivalence of (a) and (b) is evident in view of [2, pp. 42-48].

\section{BiBLIOGRAPHY}

1. J. von Neumann, Continuous geometry, (Notes by L. R. Wilcox on lectures at the Institute for Advanced Study). vol. 1, mimeographed, 1936.

2. - Continuous geometry, (Notes by L. R. Wilcox on lectures at the Institute for Advanced Study), vols. 2 and 3, planographed, Ann Arbor, Edwards Brothers, 1937.

3. - Continuous geometry, Proc. Nat. Acad. Sci., U.S.A. vol. 22 (1936) pp. 92-100.

4. - Examples of continuous geometries, Proc. Nat. Acad. Sci. U.S.A. vol. 22 (1936) pp. 101-108.

5. - On regular rings, Proc. Nat. Acad. Sci. U.S.A. vol. 22 (1936) pp. 707-713. (1937).

6. - Algebraic theory of continuous geometries, Proc. Nat. Acad. Sci. U.S.A. vol. 23

7. - Continuous rings and their arithmetics, Proc. Nat. Acad. Sci. U.S.A. vol. 23 (1937) pp. 341-349.

8. R. Baer, Linear algebra and projective geometry, New York, Academic Press, 1952.

University of Maryland,

College Park, Md.

UNIVERSITY OF TENNESSEE,

KNOXVILLE, TENN. 